\title{
Spatial-Spectral Radial Basis Function-based Interpolation for Landsat ETM+ SLC-off Image Gap Filling
}

\author{
Qunming Wang, Lanxing Wang, Zhongbin Li, Xiaohua Tong, and Peter M. Atkinson
}

\begin{abstract}
The scan-line corrector (SLC) of Landsat 7 ETM+ failed permanently in 2003, resulting in about $22 \%$ un-scanned gap pixels in the SLC-off images, affecting greatly the utility of the ETM+ data. To address this issue, we propose a spatial-spectral radial basis function (SSRBF)-based interpolation method to fill gaps in SLC-off images. Different from the conventional spatial-only radial basis function (RBF) that has been widely used in other domains, SSRBF also integrates a spectral RBF to increase the accuracy of gap filling. Concurrently, global linear histogram matching is applied to alleviate the impact of potentially large differences between the known and SLC-off images in feature space, which is demonstrated mathematically in this paper. SSRBF fully exploits information in the data themselves and is user-friendly. The experimental results on five groups of datasets covering different heterogeneous regions show that the proposed SSRBF method is an effective solution to gap filling and it can produce more accurate results than six popular benchmark methods.
\end{abstract}

Index Terms-Landsat ETM+, SLC-off, Gap filling, radial basis function (RBF) interpolation

\section{INTRODUCTION}

The Landsat series of satellites provides a valuable data source for land surface monitoring at the global scale [1]-[6], The Landsat program represents the world's longest continuous collection (from 1972 to present) of moderate spatial resolution remote sensing data [7]. Until now, the Landsat series sensors include the Landsat 1-4 Multispectral Scanners (MSS), the Landsat 5 Thematic Mapper (TM), the Landsat 7 Enhanced Thematic Mapper Plus (ETM+) and the Landsat 8 Operational Land Imager (OLI). The long-term repeat coverage [8], [9] and relatively fine spatial resolution (e.g., $30 \mathrm{~m}$ for TM, ETM+ and OLI) have led to Landsat images being one of the most used sources of global data for research and operational applications.

Amongst the Landsat series of satellites, Landsat 7 has provided high quality remote sensing data since 1999 . Although the Landsat 7 mission has exceeded its expected lifetime it is

This work was supported in part by the National Natural Science Foundation of China under Grant 41971297, in part by the Fundamental Research Funds for the Central Universities under Grant 02502150021 , and in part by the Tongji University under Grant 02502350047 (Corresponding authors: Z. Li and X. Tong).

Q. Wang, L. Wang, and X. Tong are with the College of Surveying and Geo-Informatics, Tongji University, 1239 Siping Road, Shanghai 200092, China (e-mails: wqm11111@126.com; xhtong@tongji.edu.cn).

$\mathrm{Z}$. $\mathrm{Li}$ is with the Center for Global Change and Earth Observations, Michigan State University, East Lansing, MI, USA (lzbtongji@gmail.com).

P.M. Atkinson is with the Faculty of Science and Technology, Lancaster University, Lancaster LA1 4YR, UK and also with Geography and Environment, University of Southampton, Highfield, Southampton SO17 1BJ, UK. still in operation and has become one of the most important satellites for acquiring global coverage data. However, the scan-line corrector (SLC) for the ETM+ sensor onboard Landsat 7 failed permanently on May 31, 2003. The SLC was designed to compensate for the forward motion of the satellite. As a result, un-scanned pixels (also referred to as gap pixels and missing pixels, hereafter) exist in the images acquired after the failure of the SLC. These gaps are about 12 pixels wide and occupy about $22 \%$ of the whole image. Images acquired before the failure of the SLC are usually called SLC-on images, while images acquired after the SLC failure are called SLC-off images. Fortunately, the SLC-off problem does not affect the radiometric and geometric quality of the sensor, and about $78 \%$ of the image contains effective pixels in each SLC-off acquisition [10]. Therefore, the Landsat 7 SLC-off data still have value for various applications and many users prefer to use these data rather than more expensive alternatives. However, the missing data caused by SLC-off affect greatly the application utility of Landsat 7 data and are the main obstacle to its adoption [11], [12]. To this end, it is of great value to develop approaches to fill the values (e.g., reflectance) of gap pixels. Since the Landsat 7 satellite is retiring and the Landsat 8 and Sentinel-2 satellites can also provide global coverage data, one may argue that it is not necessary to recover the gap pixels in Landsat 7 ETM+ SLC-off data. However, the Landsat 7 archive is of great significance and historical Landsat 7 time-series data (especially before the launch of Landsat 8 and Sentinel-2) can be invaluable for various applications, such as continuous monitoring of forest disturbance [13]. Moreover, the filled Landsat 7 images can be combined with Landsat 8 and Sentinel-2 images to provide a larger number of high quality images for more frequent monitoring.

Shortly after the failure of the SLC, the United States Geological Survey (USGS) published an official report indicating that the un-scanned data can be recovered using the spatially adjacent scanned data in SLC-off images, and more generally, auxiliary images (i.e., temporally close images, called known images, hereafter) [10]. This is also the basic mechanism of SLC-off image gap filling. The established gap filling methods can be grouped into two main categories according to the used data: spatial-based methods (i.e., using only spatially adjacent effective data in SLC-off images by spatial interpolation) and spatio-temporal-based methods (i.e., using also temporally neighboring known images). As a typical example of the former spatial-based methods, the plug-in of the ENVI software (called Gapfill) was developed and has been used widely as it is user-friendly and can be implemented without any auxiliary data. Although the image predicted by this 
type of method is visually complete, noticeable stripes still exist. For the latter spatio-temporal-based methods, the known images can be acquired from various sensors, including Landsat [14], [15] and non-Landsat sensors [16]-[19].

In recent years, researchers have developed a variety of methods by using effective data of both SLC-off and known images. The early methods include local linear histogram matching (LLHM) and adaptive window linear histogram matching (AWLHM). LLHM was officially released by USGS, and it is also one of the most widely used methods. In this method, one or more known images are used based on a linear transformation function established according to effective pixels in moving windows of known and SLC-off images. This category of method is simple to apply, and performs satisfactorily in homogeneous regions. On the basis of LLHM, Zhang et al. [20] proposed a method which we will refer to hereafter as the Geographically Weighted Regression (GWR) method. As an extension of LLHM, the GWR method quantifies the contributions of neighboring pixels by spatial distance weighting when predicting the values of missing pixels. Zeng et al. [21] used multi-temporal ETM+ SLC-off images to restore most of the gap pixels and applied a spatial regularization algorithm to predict the remaining gap pixels. Maxwell et al. [22] developed a multi-scale segmentation method for gap filling and this method was applied to land cover mapping [23]. Zhang et al. [24] proposed a co-kriging method to predict the gap pixels, taking the effective pixels in the SLC-off images as the principal variable and the pixels in the known images as the covariable. On this basis, Pringle et al. [25] considered the joint use of kriging and co-kriging. When there are insufficient known pixels in the neighborhood of the center gap pixel to support the application of co-kriging, the simple kriging method is adopted alternatively. The Neighborhood Similar Pixel Interpolator (NSPI) method developed by Chen et al. [26] has received increasing attention and is suitable for restoration of heterogeneous landscapes. NSPI prediction is considered as a linear superposition of predictions of spatial and temporal interpolations. Based on NSPI, the Geostatistical Neighborhood Similar Pixel Interpolator (GNSPI) was proposed to fill the gaps, in which a linear regression model is established for each land cover class and the kriging interpolation is implemented to estimate the residuals of gap pixels [27]. Luo et al. [28] predicted missing pixels by using LLHM and the $k$-means clustering algorithm jointly. Yin et al. [29] presented a direct sampling-based multiple-point geostatistical method which is simple to implement and can perform satisfactorily in heterogeneous regions. Recently, deep learning-based methods [30], [31] have also shown potential for SLC-off images gap filling.

The core of the gap filling methods is interpolation. It is undoubtedly of great interest to develop more accurate as well as simple interpolation methods for more reliable and convenient gap filling. The radial basis function (RBF) interpolation method has been acknowledged widely as a user-friendly and accurate solution due to its great non-linear modeling ability [32], [33] and it has been applied extensively in various domains [34], such as solving differential equations [35], scattered data interpolation [36], structure optimization [37] and image downscaling [38]. In this paper, for the first time, the RBF interpolation is considered for dealing with the issue of SLC-off images gap filling and, further, the conventional spatial-only RBF model is extended it for more powerful non-linear fitting (i.e., more reliable characterization of the relation between pixels in the entire image based on available spatially sparse pixels). In particular, the proposed interpolation method considers spectral information in addition to the spatial information (e.g., distance to adjacent pixels) in existing spatial-only RBF model [38]. This novel method is called spatial-spectral RBF (SSRBF) interpolation. Additionally, in some cases where rapid changes (e.g., seasonal changes) occur between the SLC-off image and its temporally closest known image, the known image may still be different from the SLC-off image in feature space, although they are very close in time. This can lead to great uncertainty in gap filling. To address this problem, global linear histogram matching (GLHM) is applied between the known and SLC-off images before gap filling. GLHM can create a new known image which is closer to the SLC-off image in feature space, thus, facilitating the post-gap filling process.

The rest of this paper is organized as follows. Section II first provides details of GLHM and the proposed SSRBF-based gap filling method. Experimental results are provided in Section III, in which the effectiveness of the proposed SSRBF method is demonstrated. Section IV further discusses potential problems and possible future directions of this research. The conclusions are drawn in Section V.

\section{METHODS}

\section{A. GLHM}

As a pre-processing step, GLHM is implemented to create a new known image, which can reduce the differences between the known image and the SLC-off image in feature space and, further, can facilitate post-gap filling. Suppose the acquisition times of the known and SLC-off images are $t_{k}$ and $t_{p}$, respectively. GLHM is performed by constructing a linear regression model according to the effective pixels of the known and SLC-off images

$$
L\left(x, y, b, t_{p}\right)=A_{b} \times L\left(x, y, b, t_{k}\right)+B_{b}+\gamma
$$

where $L\left(x, y, b, t_{k}\right)$ and $L\left(x, y, b, t_{p}\right)$ are values (i.e., reflectances in this paper) of the pixels located at $(x, y)$ in band $b$ of the known and SLC-off images, respectively. $A_{b}$ and $B_{b}$ are two coefficients, which can be estimated by least squares fitting according to the set of effective pixels. $\gamma$ is the residual of the regression model. The two coefficients are then used to transform the known image to a new image $\mathbf{L}^{\prime}$. The new known image is closer to the SLC-off image in feature space, as demonstrated below.

We prove the effectiveness of GLHM by mathematical derivation. For brevity, the following formulas are based on a single band of the images.

First, a linear regression model is constructed according to the effective pixels of the known and SLC-off images acquired at $t_{k}$ and $t_{p}$, respectively, as

$$
\mathbf{L}_{p}=A \mathbf{L}_{k}+B+\gamma
$$


where $\mathbf{L}_{p}$ and $\mathbf{L}_{k}$ are the reflectance matrices of the SLC-off and known images, respectively. $A$ and $B$ are two coefficients, which can be estimated by least squares fitting. $\gamma$ is the residual image of the regression model. Based on the relation characterized by the model, the difference $\Delta \mathbf{L}$ between the effective pixels of images acquired at $t_{k}$ and $t_{p}$ is defined as

$$
\begin{aligned}
\Delta \mathbf{L} & =\mathbf{L}_{p}-\mathbf{L}_{k} \\
& =(A-1) \mathbf{L}_{k}+B+\boldsymbol{\gamma}
\end{aligned} .
$$

After GLHM, the new known image $\mathbf{L}_{k}^{\prime}$ is created by a linear transformation of the original known image $\mathbf{L}_{k}$ using the coefficients $A$ and $B$

$$
\mathbf{L}_{k}^{\prime}=A \mathbf{L}_{k}+B \text {. }
$$

Thus, the difference $\Delta \mathbf{L}^{\prime}$ between the SLC-off image and the known image after GLHM is defined as

$$
\Delta \mathbf{L}^{\prime}=\mathbf{L}_{p}-\mathbf{L}_{k}^{\prime}=\boldsymbol{\gamma} .
$$

To compare the two difference images quantitatively, the expectations of the squares of $\Delta \mathbf{L}$ and $\Delta \mathbf{L}^{\prime}$ are calculated, respectively.

1) $E\left(\Delta \mathbf{L}^{2}\right)$ without GLHM

According to the relation between the expectation and the variance, we have

$$
E\left(\Delta \mathbf{L}^{2}\right)=\operatorname{Var}(\Delta \mathbf{L})+E^{2}(\Delta \mathbf{L}) .
$$

The first term in Eq. (6), by substituting Eq. (3) and considering $B$ is a constant, can be transformed based on the basic property of the variance

$$
\begin{aligned}
\operatorname{Var}(\Delta \mathbf{L}) & =\operatorname{Var}\left[(A-1) \mathbf{L}_{k}+\boldsymbol{\gamma}\right] \\
& =\operatorname{Var}\left[(A-1) \mathbf{L}_{k}\right]+\operatorname{Var}(\boldsymbol{\gamma})+2(A-1) \operatorname{Cov}\left(\mathbf{L}_{k} \cdot \boldsymbol{\gamma}\right)
\end{aligned}
$$

where the operator " ' represents the inner product between two matrices. The term $\operatorname{Cov}\left(\mathbf{L}_{k} \cdot \boldsymbol{\gamma}\right)$ in Eq. (7) can be expanded according to the basic property of the covariance

$$
\operatorname{Cov}\left(\mathbf{L}_{k} \cdot \boldsymbol{\gamma}\right)=E\left(\mathbf{L}_{k} \cdot \boldsymbol{\gamma}\right)-E\left(\mathbf{L}_{k}\right) E(\boldsymbol{\gamma}) \text {. }
$$

For the classical least squares linear regression model, there are two important properties: 1) the expectation of the residual is zero and 2) the expectation of the product of the independent variable and the residual is zero

$$
\left\{\begin{array}{c}
E(\boldsymbol{\gamma})=0 \\
E\left(\mathbf{L}_{k} \cdot \boldsymbol{\gamma}\right)=0
\end{array} .\right.
$$

Thus, Eq. (8) equals zero according to Eq. (9), and Eq. (7) can be simplified further as

$$
\operatorname{Var}(\Delta \mathbf{L})=\operatorname{Var}\left[(A-1) \mathbf{L}_{k}\right]+\operatorname{Var}(\gamma) .
$$

As a result, Eq. (6) can be expressed as

$$
E\left(\Delta \mathbf{L}^{2}\right)=\operatorname{Var}\left[(A-1) \mathbf{L}_{k}\right]+\operatorname{Var}(\boldsymbol{\gamma})+E^{2}(\Delta \mathbf{L}) .
$$

2) $E\left[\left(\Delta \mathbf{L}^{\prime}\right)^{2}\right]$ after GLHM

It can be seen from Eq. (5) that $E\left[\left(\Delta \mathbf{L}^{\prime}\right)^{2}\right]=E\left(\gamma^{2}\right)$. According to the relation between the expectation and the variance

$$
E\left(\gamma^{2}\right)=\operatorname{Var}(\gamma)+E^{2}(\gamma) .
$$

Based on Eq. (9), the second term in Eq. (12) equals zero. Therefore, Eq. (12) can be simplified as

$$
E\left(\gamma^{2}\right)=\operatorname{Var}(\gamma) \text {. }
$$

Comparing Eq. (11) with Eq. (13), it is seen clearly that $E\left[\left(\Delta \mathbf{L}^{\prime}\right)^{2}\right]<E\left(\Delta \mathbf{L}^{2}\right)$ (the first and third terms in Eq. (11) are larger than zero). In conclusion, it is proved that compared with the original known image, the new known image after GLHM is closer to the SLC-off image in feature space.

\section{B. $S S R B F$}

In this paper, the SSRBF method is proposed to predict the missing pixels. Different from the conventional spatial-based RBF, SSRBF considers additional spectral information for enhancement. As the relation between pixels decreases or even disappears with increasing separation distance, a fixed-size moving window (e.g., a $35 \times 35$ pixel window in this paper) is considered. Note that the known image mentioned below is the image created by GLHM. The implementation of SSRBF is illustrated as follows:

1) Selection of neighboring similar pixels. For each gap pixel, its neighboring pixels are used to predict the reflectance, as they are closely related to the center pixel. Taking land cover heterogeneity into consideration, however, many neighboring pixels may belong to different land cover classes from the center pixel and, thus, have substantially different spectra. Thus, it is logical to select neighboring pixels with similar spectra to the center pixel for gap filling. Because the reflectance of the center gap pixel is unknown, the known image is used to select similar pixels. We assume that no rapid land cover changes occurred between the acquisition times of the known and SLC-off images, and the similar pixels selected from the known image are also spectrally similar to the center gap pixel in the SLC-off image. The spectral similarity is quantified using the root mean square deviation (RMSD) [25]

$$
R M S D_{i j}=\sqrt{\frac{\sum_{b=1}^{n}\left[L\left(x_{i}, y_{i}, b, t_{k}\right)-L\left(x_{j}, y_{j}, b, t_{k}\right)\right]^{2}}{n}}
$$

where $L\left(x_{i}, y_{i}, b, t_{k}\right)$ and $L\left(x_{j}, y_{j}, b, t_{k}\right)$ are reflectances of the $i$ - and $j$-th pixel in band $b$ of the known image, and $n$ is the number of spectral bands. A small RMSD denotes a small spectral difference. According to Eq. (14), the RMSDs between a center pixel and its neighboring pixels in the moving window of the known image are calculated and then the $N$ pixels with the smallest values are identified as similar pixels.

2) Construction of spatial-based RBF. The widely used Gaussian function is considered to define the spatial-based RBF $\varphi_{D i j}$

$$
\varphi_{D i j}=e^{-\sqrt{\frac{\left(x_{i}-x_{j}\right)^{2}+\left(y_{i}-y_{j}\right)^{2}}{\delta_{1}}}} .
$$

In Eq. (15), $\left(x_{i}, y_{i}\right)$ and $\left(x_{j}, y_{j}\right)$ are the locations of the $i$ - and $j$-th spectrally similar pixels in the local window and $\delta_{1}$ is a parameter. In this paper, the parameter for the spatial-based $\mathrm{RBF}$ is determined as twice the maximum distance between the center pixel and the neighboring pixels in the window. Specifically, the maximum distance is $17 \sqrt{2}$ when the size of the window is $35 \times 35$, and in this case, $\delta_{1}$ can be approximately determined as 50 . 
Furthermore, the spatial-based $\mathrm{RBF} \varphi_{D i}$ between the center and similar pixels is defined as

$$
\varphi_{D i}=e^{-\sqrt{\frac{\left(x_{i}-x_{0}\right)^{2}+\left(y_{i}-y_{0}\right)^{2}}{\delta_{1}}}}
$$

where $\left(x_{0}, y_{0}\right)$ indicates the location of the center pixel.

3) Construction of SSRBF. It is seen from Eqs. (15) and (16) that the spatial-based RBF simply considers the spatial distance between pixels, that is, the larger the distance, the smaller the value of the spatial-based RBF (i.e., smaller spatial relation between the two pixels). However, it may not be sufficient to characterize the relation between pixels based solely on spatial distance, as the reflectances of some pixels can be very different even they are spatially close and also some pixels may be very similar even they are spatially distant. Thus, when characterizing the RBF, it is helpful to account for the spectral information in addition to spatial distance as in the spatial-only RBF. Accordingly, the spectral-based $\operatorname{RBF} \varphi_{R i j}$ between similar pixels and $\varphi_{R i}$ between the center pixel and its neighboring similar pixels are defined as

$$
\left\{\begin{array}{l}
\varphi_{R i j}=e^{-\frac{R M S D_{i j}}{\delta_{2}}} \\
\varphi_{R i}=e^{-\frac{R M S D_{i}}{\delta_{2}}}
\end{array}\right.
$$

where $R M S D_{i}$ is the RMSD between the center pixel and the $i$-th similar pixel in the window and $\delta_{2}$ is the parameter of the spatial-based RBF. The parameter $\delta_{2}$ is determined according to the set of RMSD values for all windows. Specifically, if almost all RMSD values are smaller than 0.05 , then $\delta_{2}$ is set to 0.1 , which is twice the maximum value. A larger RMSD leads to a smaller value of the spatial-based RBF, indicating a smaller correlation between pixels.

Based on the definitions of the spatial- and spectral-based RBF, the SSRBF is proposed by integrating both terms. This characterizes the relation between pixels more reliably by considering the spatial and spectral information simultaneously and exploiting fully the information in the data themselves. Correspondingly, the SSRBF between similar pixels (denoted as $\left.\varphi_{i j}\right)$ and between the center pixel and its neighboring similar pixels (denoted as $\varphi_{i}$ ) are defined as

$$
\left\{\begin{array}{c}
\varphi_{i j}=\varphi_{D i j} \varphi_{R i j} \\
\varphi_{i}=\varphi_{D i} \varphi_{R i}
\end{array} .\right.
$$

4) SSRBF for gap filling. It is acknowledged widely in the existing literature [25], [26], [39], [40] that the center gap pixel is always expected to have similar temporal changes to its neighboring similar pixels. In this paper, SSRBF is proposed to estimate the temporal changes in reflectance for gap pixels. The SSRBF prediction for each gap pixel is a linear combination of $\varphi_{i}$ for all its similar pixels and the key is the calculation of the weights through effective pixels. Specifically, the temporal changes between the known and SLC-off images for the $N$ similar pixels are first calculated as $\Delta L\left(x_{i}, y_{i}, b\right)=L\left(x_{i}, y_{i}, b, t_{p}\right)-L^{\prime}\left(x_{i}, y_{i}, b, t_{k}\right)$. The weights, denoted as $\omega_{b i}(i=1,2, \ldots, N)$, can then be calculated from the following equation:

$$
\left[\begin{array}{ccccc}
\varphi_{11} & \cdots & \varphi_{1 i} & \cdots & \varphi_{1 N} \\
\vdots & \cdots & \vdots & \cdots & \vdots \\
\varphi_{i 1} & \cdots & \varphi_{i i} & \cdots & \varphi_{i N} \\
\vdots & \cdots & \vdots & \cdots & \vdots \\
\varphi_{N 1} & \cdots & \varphi_{N i} & \cdots & \varphi_{N N}
\end{array}\right]\left[\begin{array}{c}
\omega_{b 1} \\
\vdots \\
\omega_{b i} \\
\vdots \\
\omega_{b N}
\end{array}\right]=\left[\begin{array}{c}
\Delta L\left(x_{1}, y_{1}, b\right) \\
\vdots \\
\Delta L\left(x_{i}, y_{i}, b\right) \\
\vdots \\
\Delta L\left(x_{N}, y_{N}, b\right)
\end{array}\right]
$$

For each gap pixel, a unique set of weights $\omega_{b i}$ is produced with Eq. (19) and its temporal change $\Delta L\left(x_{0}, y_{0}, b\right)$ is estimated as

$$
\Delta L\left(x_{0}, y_{0}, b\right)=\sum_{i=1}^{N} \omega_{b i} \varphi_{i} .
$$

The final prediction is the combination of the known image produced by GLHM and the predicted temporal change

$$
\hat{L}\left(x_{0}, y_{0}, b, t_{p}\right)=L^{\prime}\left(x_{0}, y_{0}, b, t_{k}\right)+\Delta L\left(x_{0}, y_{0}, b\right) .
$$

The overview of the implementation of the proposed method is shown in Fig. 1.

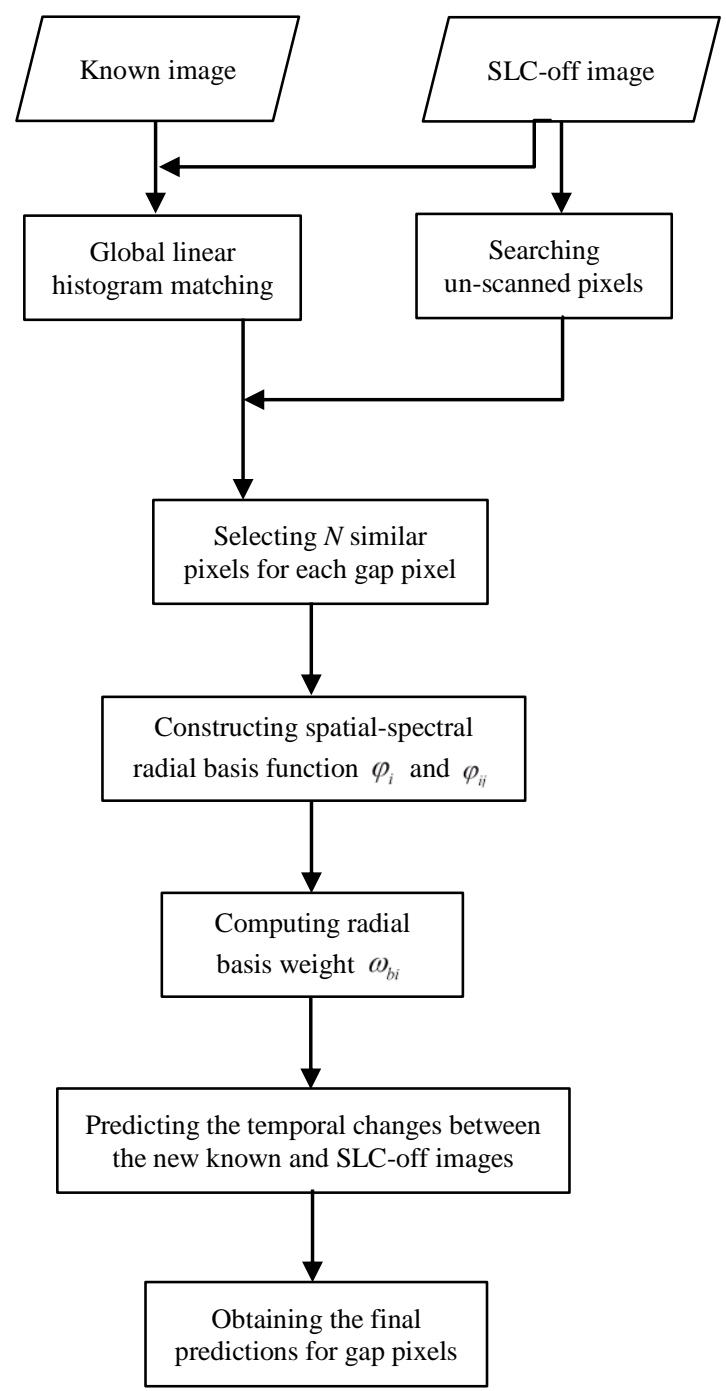

Fig. 1. Flowchart of the proposed SSRBF-based gap filling method. 


\section{EXPERIMENTS}

\section{A. Data and experimental design}

The proposed SSRBF method was validated with both simulated and real ETM+ SLC-off images, and five groups of datasets covering different regions were used in the experiments. The five regions are located in Verona, Italy (Region 1), Zhejiang Province, China (Region 2), Northern New South Wales, Australia (Region 3), Beijing, China (Region 4) and Versailles, France (Region 5). Regions $1-3$ cover $15 \mathrm{~km} \times 15 \mathrm{~km}$ (500 $\times 500$ Landsat pixels) areas, while Regions 4 and 5 both cover $36 \mathrm{~km} \times 36 \mathrm{~km}(1200 \times 1200$ Landsat pixels $)$ areas. The data used include three Landsat 8 OLI images for Region 1, two Landsat 8 OLI images for Region 2 and 3, as well as one Landsat 7 ETM+ SLC-off and one Landsat 8 OLI images for both Regions 4 and 5, as shown in Table 1.

It should be noted that for Regions 1-3, the Landsat 8 OLI images were used in the simulation experiments in place of real Landsat 7 ETM+ SLC-off images. That is, the ETM+ SLC-off images were simulated using the Landsat 8 OLI images. This is because there are no reference data of the gaps for objective evaluation, if real SLC-off images are used. On the contrary, the corresponding data in the simulated ETM+ SLC-off images are known perfectly for both visual and quantitative evaluation. Moreover, the Landsat 8 OLI bands also have the same wavelengths as the Landsat 7 ETM+ bands. Thus, the scheme of simulating ETM+ SLC-off images using OLI images can persuasively reflect the performances of the gap filling methods. On the other hand, to further show the performance of the SSRBF-based gap filling method in real cases, the real Landsat 7 ETM+ SLC-off images were also involved in the later experiments on Regions 4 and 5 in this paper.

Table 1 Images used in the experiments ( $\mathrm{S}$ represents the Landsat 8 OLI image used to simulate the SLC-off image, L represents the Landsat 8 OLI data used as known images and R represents the real Landsat 7 ETM+ SLC-off images)

\begin{tabular}{|c|c|c|}
\hline Region & Number & Acquisition date \\
\hline \multirow{2}{*}{$\begin{array}{c}\text { Verona, Italy } \\
\text { (Region 1; heterogeneous) }\end{array}$} & $\mathrm{S} 1$ & 2016.08 .27 \\
\cline { 2 - 3 } & $\mathrm{L} 11$ & 2016.10 .30 \\
\hline Zhejiang, China & L12 & 2017.01 .02 \\
\hline $\begin{array}{c}\text { Zhej } \\
\text { (Region 2; heterogeneous) }\end{array}$ & $\mathrm{S} 2$ & 2016.04 .22 \\
\cline { 2 - 3 } $\begin{array}{c}\text { Northern New South Wales, Australia } \\
\text { (Region 3; abrupt change) }\end{array}$ & $\mathrm{S} 3$ & 2016.07 .20 \\
\cline { 2 - 3 } & $\mathrm{L} 3$ & 2004.12 .12 \\
\hline
\end{tabular}

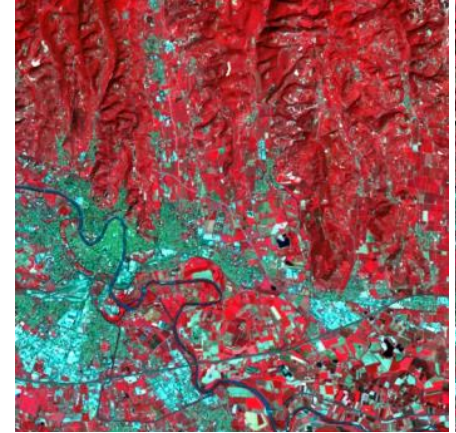

(a)

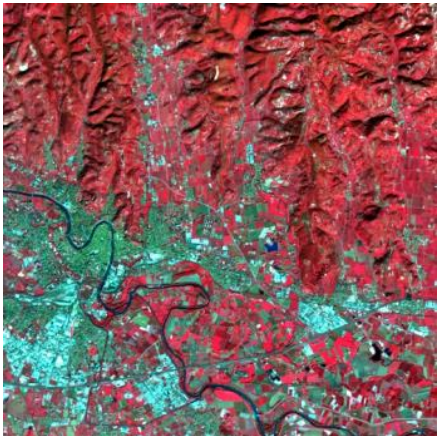

(b)

\begin{tabular}{|c|c|c|}
\hline Beijing, China & R4 & 2019.05 .21 \\
\cline { 2 - 3 } (Region 4; heterogeneous) & L4 & 2019.03 .26 \\
\hline Versailles, France & R5 & 2020.05 .27 \\
\cline { 2 - 3 } (Region 5; abrupt change) & L5 & 2020.08 .07 \\
\hline
\end{tabular}

In the simulation experiments on Regions 1-3, the Landsat 8 OLI images acquired on August 27, 2016 (S1), April 22, 2016 (S2) and December 12, 2004 (S3) were used to simulate the SLC-off images, respectively. The images acquired on other dates were used as the known images for gap filling. Finally, the original gap-free images S1, S2 and S3 were used for evaluation. Figs. 2-6 show the images for Regions 1-5, respectively. Region 1 is a typical rural area in which the crops show different seasonal changes, resulting in great heterogeneity. From Fig. 2, it can be seen that S1 and L11 are similar in hue while L12 is less similar to them mainly due to the distinct difference between the vegetation in autumn (Fig. 2(a) and (b)) and winter (Fig. 2(c)). Region 2 is a typical urban area, and similar changes in hue can also be observed in Fig. 3. Moreover, most of the buildings in Fig. 3 have smaller spatial width than the gaps. Thus, the ability of each method to restore the spatial details can be examined. In Fig. 4, we can see the abrupt changes caused by flood inundation in Region 3, which was a challenge for gap filling. Region 4 represents a real case experiencing significant seasonal changes in urban areas. Region 5 is dominated by farmlands with a wide range of significant changes (caused by crop rotation) between the SLC-off and known images.

The proposed SSRBF-based gap filling method is compared with several benchmark methods in the simulation experiments on Regions 1-3, including Gapfill, LLHM, GWR, weighted multiple linear regression (WMLR) [41], NSPI and GNSPI. We set a fixed size of $35 \times 35$ pixels for the moving window and the same number of similar pixels of 20 for all methods. It should be emphasized that regarding the choice of number of similar pixels, 20 has been widely selected, such as in the NSPI and GNSPI. Specifically, the accuracy of gap filling tends to be stable when the number exceeds 20 , but the computing time increases obviously as the number increases further. That is, the number of 20 balances satisfactorily model accuracy and computational burden. For the experiments on real ETM+ SLC-off images in Regions 4 and 5, the performance of SSRBF is examined by visual inspection.

Fig. 2. Landsat 8 OLI data used in the simulation experiment for Region 1 (NIR, red and green bands as RGB). (a) S1. (b) L11. (c) L12. 


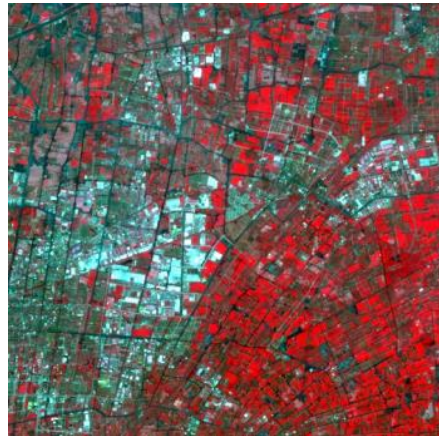

(a)

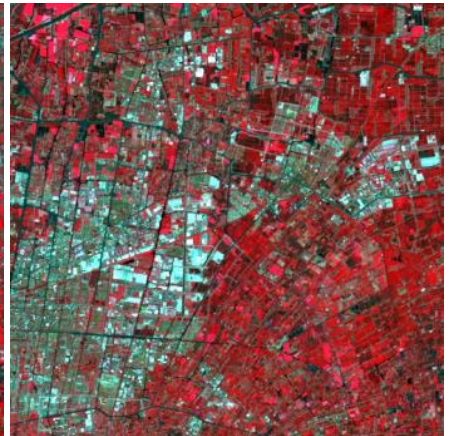

(b)
Fig. 3. Landsat 8 OLI data used in the simulation experiment for Region 2 (NIR, red and green bands as RGB). (a) S2. (b) L2.

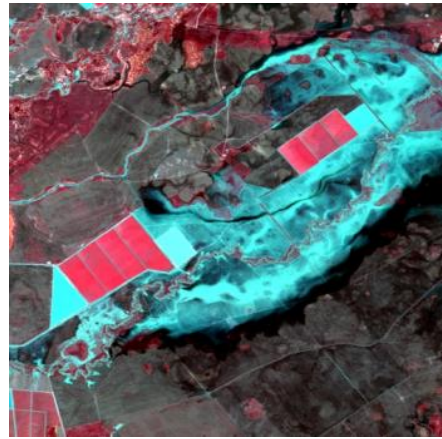

(a)

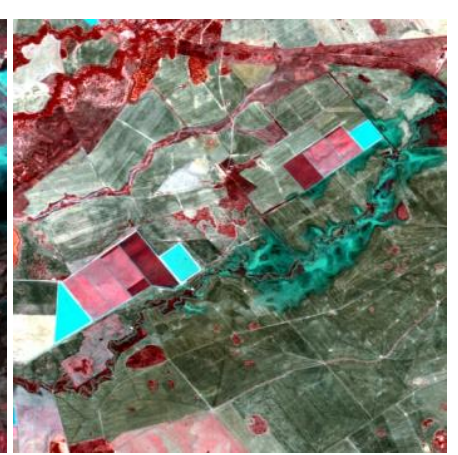

(b)
Fig. 4. Landsat 8 OLI data used in the simulation experiment for Region 3 (NIR, red and green bands as RGB). (a) S3. (b) L3.

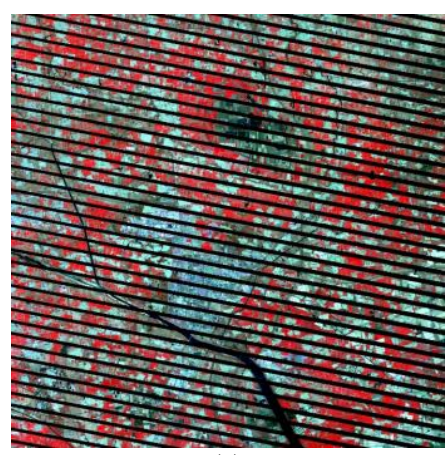

(a)

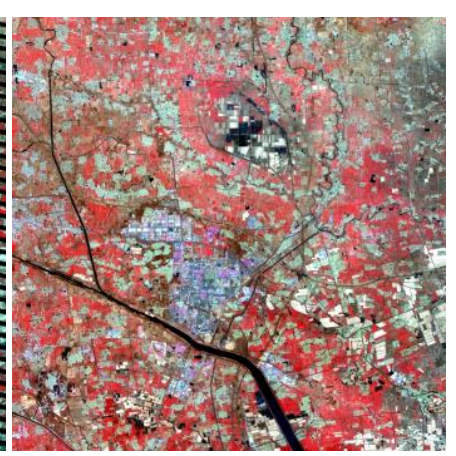

(b)
Fig. 5. Landsat 7 ETM+ SLC-off and Landsat 8 OLI data used in the experiment for Region 4 (NIR, red and green bands as RGB). (a) R4. (b) L4.

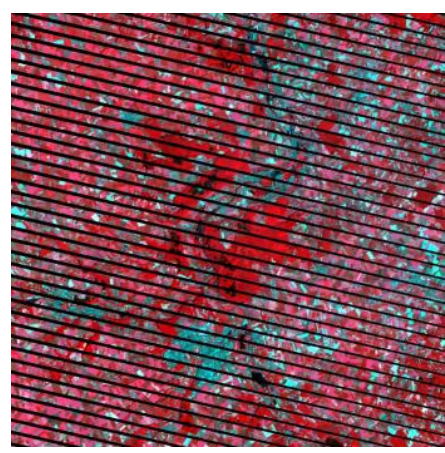

(a)

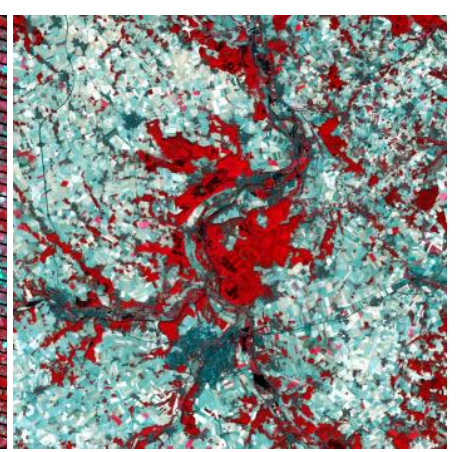

(b)
Fig. 6. Landsat 7 ETM+ SLC-off and Landsat 8 OLI data used in the experiment for Region 5 (NIR, red and green bands as RGB). (a) R5. (b) L5.

\section{B. Simulation experiment on the Verona data (Region 1; heterogeneous)}

In the experiment on Region 1, the SLC-off image simulated based on S1 (acquired on August 27, 2016) is shown in Fig. 7(a). L11 and L12 were used as the input known images and the gap-free S1 was used for evaluation. Fig. 7(b)-Fig. 7(h) show the gap filling results using L12 in Fig. 2(c) as the known image based on the Gapfill, LLHM, GWR, WMLR, NSPI, GNSPI and SSRBF methods, respectively. It is obvious that all filled images are similar to the actual image to various extents. Amongst them, there are apparent stripes in predictions of the Gapfill, LLHM, GWR and WMLR methods, while the predictions produced by NSPI, GNSPI and SSRBF are much closer to the actual image. To show the differences between the methods more clearly, two subareas are selected for each image, which are enlarged and placed on the right side of each entire image. The results show that Gapfill produces ambiguous artifacts in both subareas and cannot restore the spatial details of objects satisfactorily. For example, the contour of the red object at the center of the first subarea is not restored, and the red object on the right in the second subarea is partially predicted as yellow. The results of LLHM and GWR are very similar: the red part of the central object in the first subarea is predicted as green which is similar to the surrounding features, and the yellow feature in the second subarea is incorrectly predicted as light red, showing large stripes. A small part of the red object predicted by WMLR in the first subarea is close to the reference, but the other pixels are obviously different from the real data. In the second subarea of the WMLR result, the prediction is visually more accurate than Gapfill, LLHM and GWR, but the color of some pixels is inappropriately predicted as red. For the NSPI and GNSPI results, the red object in the center of the first subarea is partly predicted as small green patches; in the second subarea, there are some dark pixels on the left side of the yellow object. Compared with the six benchmark methods, SSRBF produces a result closer to the actual image (see, e.g., restoration of the red and yellow objects in the first and second subareas, respectively). The same conclusion can also be drawn when L11 was used as the known image, as shown in the results in Fig. 8.

To observe the difference between the NSPI and SSRBF predictions more clearly, the error images of both methods for the two subareas in Fig. 7 are shown in Figs. 9 and 10. The error image of each band is composed of the absolute value of the difference between the true and predicted reflectances, in which the value of zero is set to black. From Figs. 9 and 10, two conclusions can be drawn. First, the error of the filling results (including the results of both NSPI and SSRBF) is more obvious near the center of the stripe. Second, the error of SSRBF is obviously smaller than of NSPI. Taking the results of the NIR band in Fig. 9 as an example, in the middle of the stripe, the error map of SSRBF is generally blue with very few red pixels while the error map of NSPI is basically yellow or even red in the central region. In Fig. 10, a similar conclusion can be drawn for all bands. Note that for the NIR band in Fig. 10, while SSRBF produces larger errors for several pixels on the left than that for the NSPI, the errors are smaller for the middle pixels.

The scatter plots can reveal the difference between the actual and the predicted reflectances intuitively. Thus, the scatter plots between the predictions in Fig. 7 and the reference are shown in 
Fig. 11, where the NIR band is taken as an example. It is seen that the points of the SSRBF method are more aggregated and closer to the diagonal line, while the points of other methods present greater dispersion. This means that even though the
SSRBF prediction produces larger errors in a few local areas (as shown in the NIR band in Fig. 10), its overall error is smaller. That is, the prediction of the proposed SSRBF method is in greater agreement with the actual image.

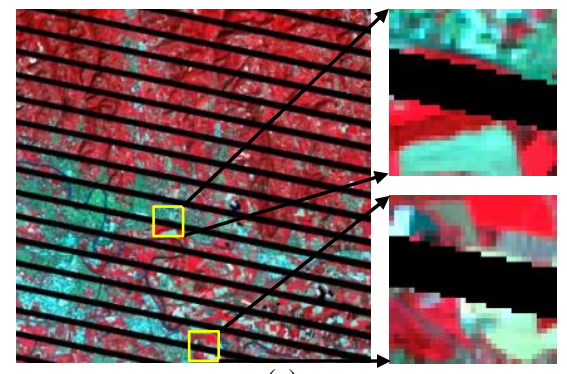

(a)

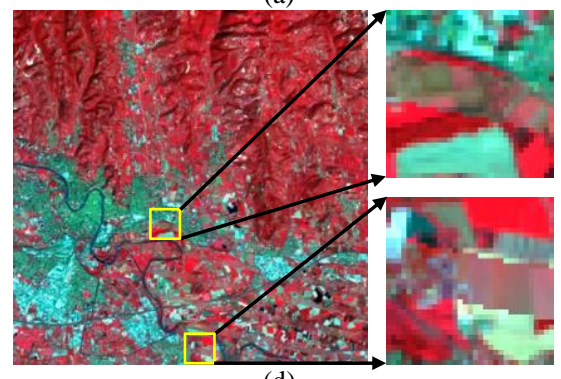

(d)

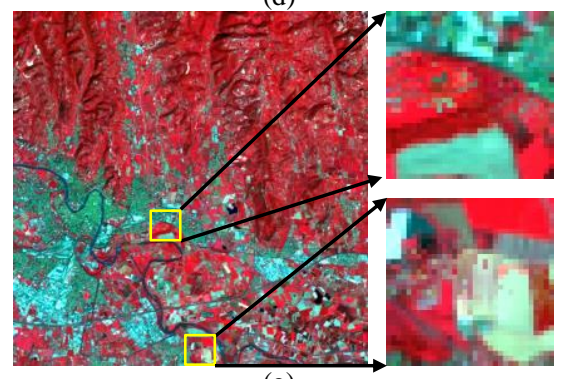

(g)



(b)

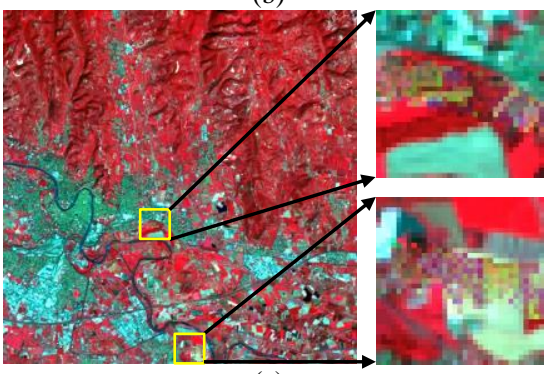

(e)

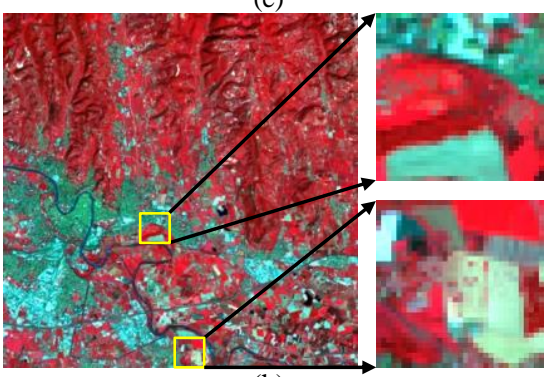

(h)

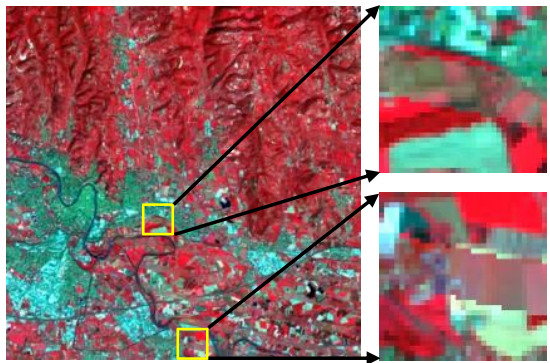

(c)

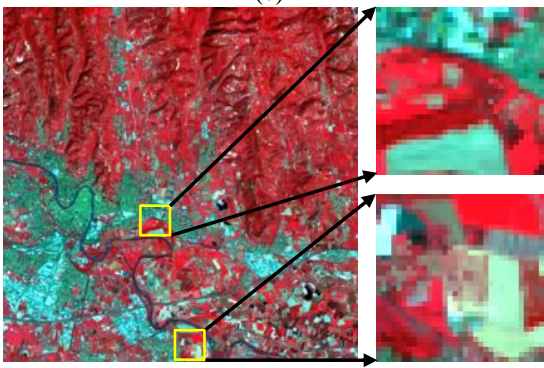

(f)

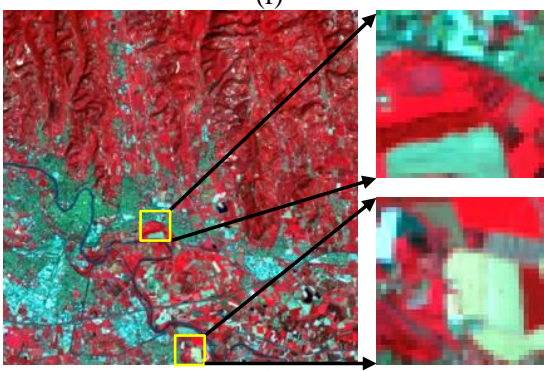

(i)

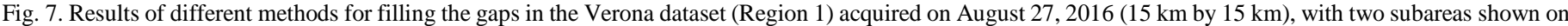

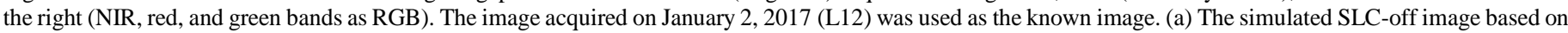
(g); (b) Gapfill. (c) LLHM. (d) GWR. (e) WMLR. (f) NSPI. (g) GNSPI. (h) SSRBF. (i) The actual image.

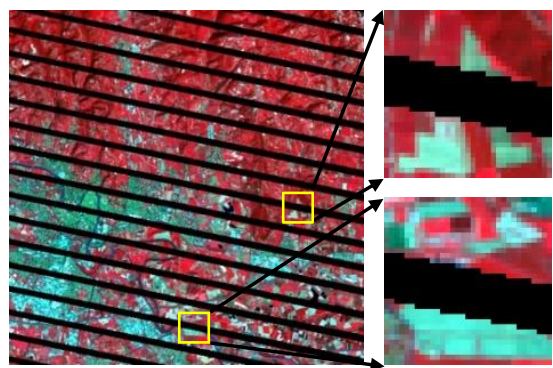

(a)

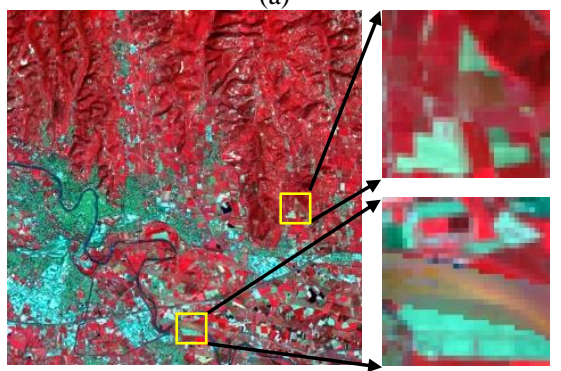

(d)

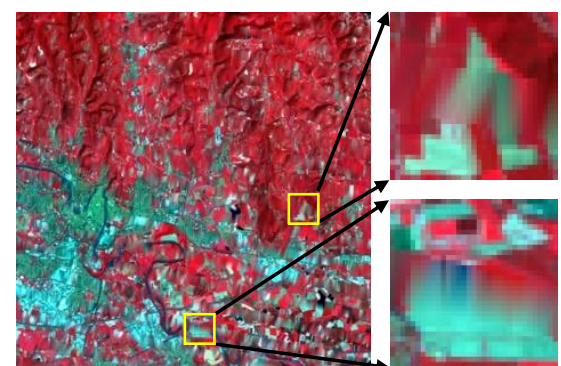

(b)

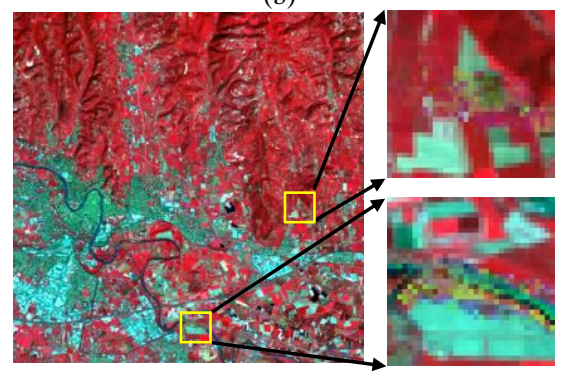

(e)

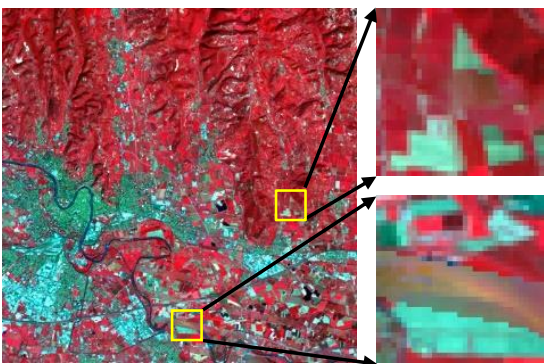

(c)

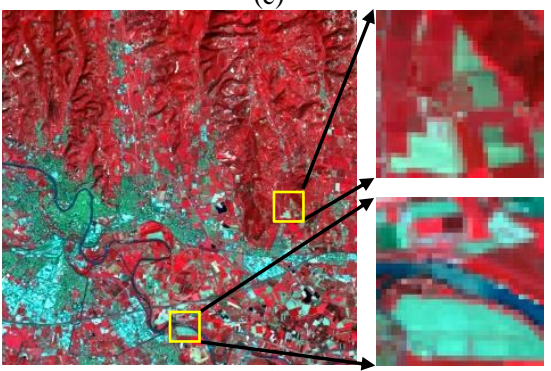

(f) 


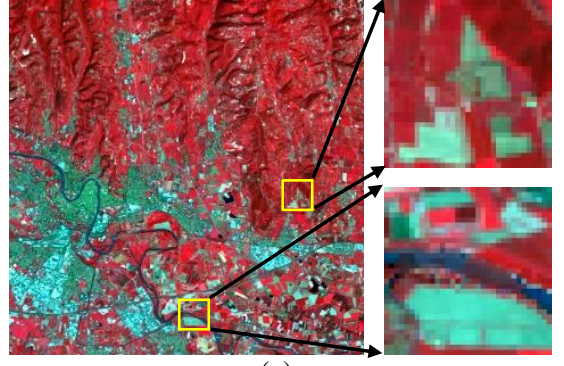

(g)

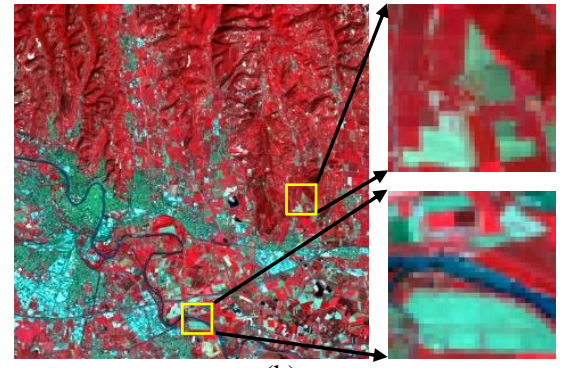

(h)

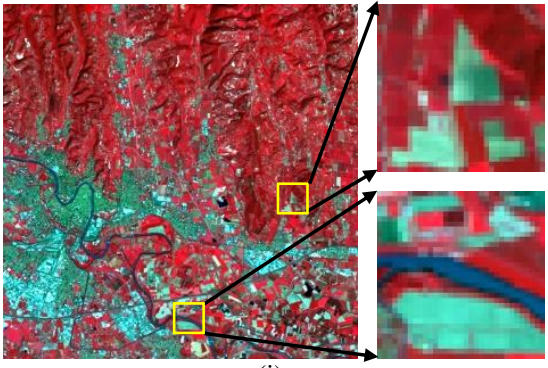

(i)

Fig. 8. Results of different methods for filling the gaps in the Verona dataset (Region 1) acquired on August 27, 2016 (15 km by $15 \mathrm{~km}$ ), with two subareas shown on the right (NIR, red, and green bands as RGB). The image acquired on October 30, 2016 (L11) was used as the known image. (a) The simulated SLC-off image based on (i); (b) Gapfill. (c) LLHM. (d) GWR. (e) WMLR. (f) NSPI. (g) GNSPI. (h) SSRBF. (i) The actual image.
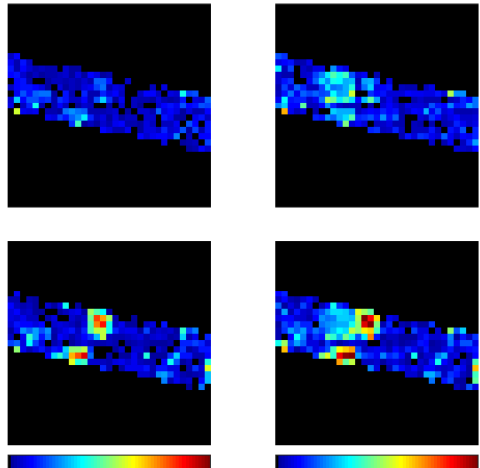

0

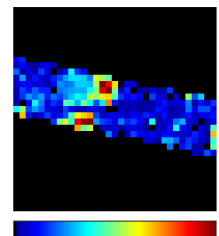

$0.05 \quad 0$
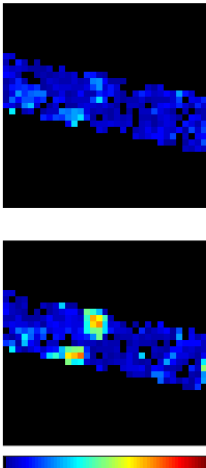

$0.15 \quad 0$
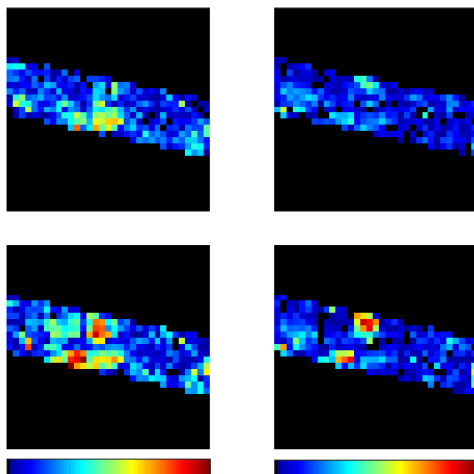

0.250

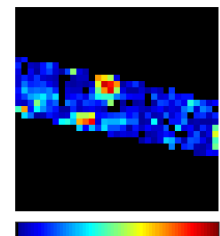

$0.15 \quad 0$
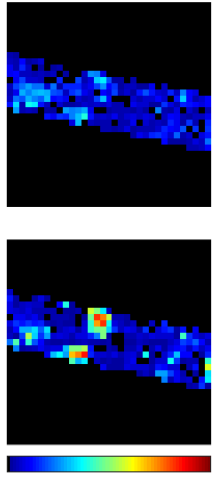

0.2

Fig. 9. Error images of SSRBF (top) and NSPI (bottom) for the first subarea in Fig. 7. Bands from left to right are blue, green, red, NIR, SWIR1 and SWIR2.
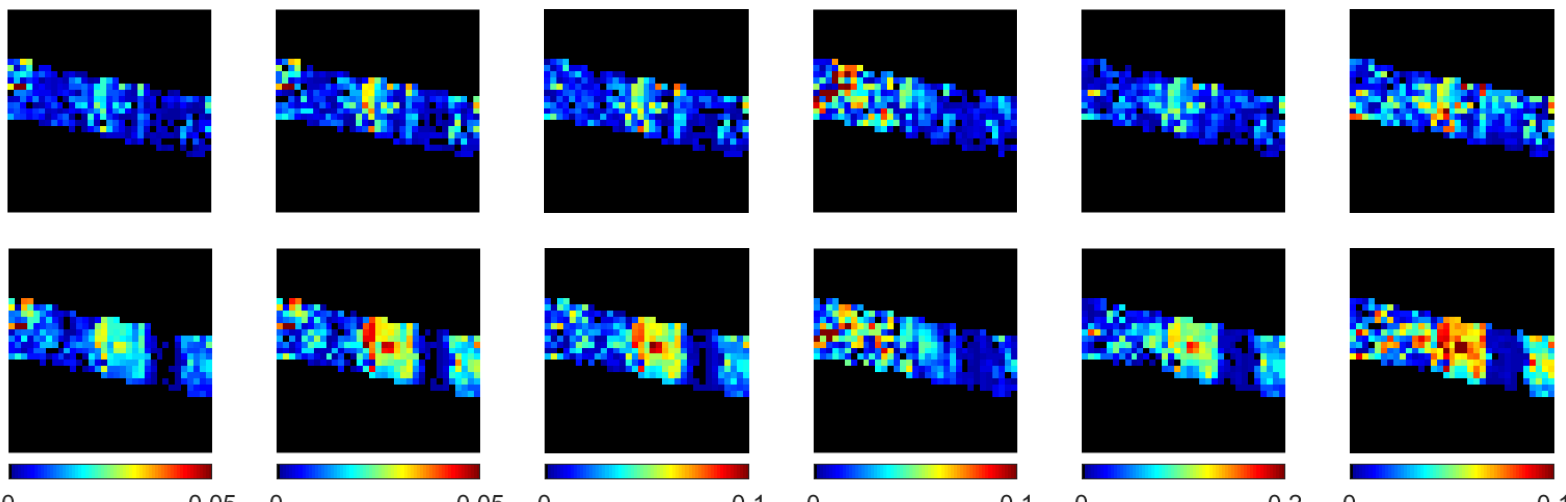

$0.05 \quad 0$

$0.05 \quad 0$

0.10

0.10

0.2

Fig. 10. Error images of SSRBF (top) and NSPI (bottom) for the second subarea in Fig. 7. Bands from left to right are blue, green, red, NIR, SWIR1 and SWIR2.

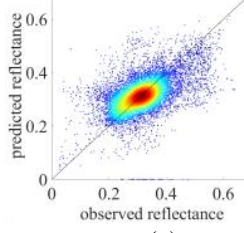

(a)

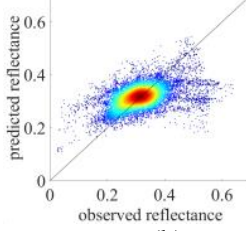

(b)

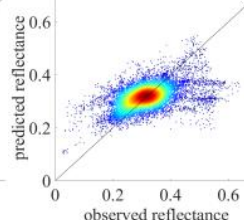

(c)

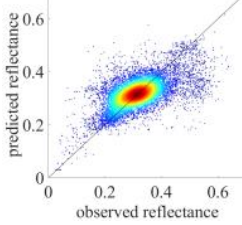

(d)

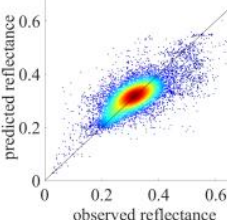

(e)

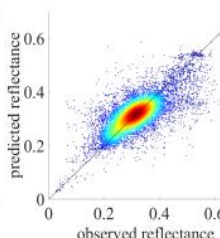

(f)

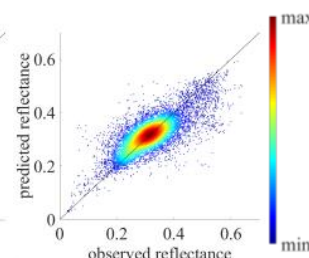

(g)

Fig. 11. Scatter plots between the actual and predicted reflectances of the NIR band for the results in Fig. 7. (a) Gapfill. (b) LLHM. (c) GWR. (d) WMLR. (e) NSPI. (f) GNSPI. (g) SSRBF.

Table 2 Accuracies of the gap filling methods produced using different known images (L11 and L12) in Verona, Italy (Region 1) (the values in bold and italic mean the most, and second most, accurate results in each case)

\begin{tabular}{|c|c|c|c|c|c|c|c|c|c|}
\hline & & & Blue & Green & Red & NIR & SWIR1 & SWIR2 & Mean \\
\hline \multirow{6}{*}{$\begin{array}{c}\text { L11 as } \\
\text { known } \\
\text { image }\end{array}$} & \multirow{6}{*}{ RMSE } & Gapfill & 0.0179 & 0.0222 & 0.0318 & 0.0592 & 0.0503 & 0.0462 & 0.0379 \\
\hline & & LLHM & 0.0102 & 0.0140 & 0.0224 & 0.0506 & 0.0394 & 0.0374 & 0.0290 \\
\hline & & GWR & 0.0102 & 0.0140 & 0.0224 & 0.0503 & 0.0392 & 0.0373 & 0.0289 \\
\hline & & WMLR & 0.0099 & 0.0135 & 0.0212 & 0.0462 & 0.0368 & 0.0357 & 0.0272 \\
\hline & & NSPI & 0.0090 & 0.0120 & 0.0187 & 0.0407 & 0.0332 & 0.0313 & 0.0241 \\
\hline & & GNSPI & 0.0095 & 0.0127 & 0.0194 & 0.0412 & 0.0349 & 0.0334 & 0.0252 \\
\hline
\end{tabular}




\begin{tabular}{|c|c|c|c|c|c|c|c|c|c|}
\hline & & SSRBF & 0.0086 & 0.0116 & 0.0179 & 0.0388 & 0.0323 & 0.0302 & 0.0232 \\
\hline & \multirow{7}{*}{$\mathrm{CC}$} & Gapfill & 0.7241 & 0.6815 & 0.6882 & 0.6551 & 0.5212 & 0.6025 & 0.6454 \\
\hline & & LLHM & 0.9168 & 0.8826 & 0.8527 & 0.7452 & 0.7231 & 0.7506 & 0.8118 \\
\hline & & GWR & 0.9172 & 0.8834 & 0.8541 & 0.7482 & 0.7254 & 0.7531 & 0.8136 \\
\hline & & WMLR & 0.9214 & 0.8905 & 0.8700 & 0.7936 & 0.7629 & 0.7767 & 0.8358 \\
\hline & & NSPI & 0.9367 & 0.9156 & 0.9004 & 0.8442 & 0.8127 & 0.8337 & 0.8739 \\
\hline & & GNSPI & 0.9276 & 0.9044 & 0.8917 & 0.8415 & 0.7909 & 0.8081 & 0.8607 \\
\hline & & SSRBF & 0.9408 & 0.9207 & 0.9095 & 0.8599 & 0.8237 & 0.8460 & 0.8834 \\
\hline & \multirow{7}{*}{ UIQI } & Gapfill & 0.7163 & 0.6701 & 0.6766 & 0.6463 & 0.4996 & 0.5837 & 0.6321 \\
\hline & & LLHM & 0.9103 & 0.8711 & 0.8388 & 0.7190 & 0.6822 & 0.7178 & 0.7899 \\
\hline & & GWR & 0.9108 & 0.8719 & 0.8402 & 0.7221 & 0.6849 & 0.7204 & 0.7917 \\
\hline & & WMLR & 0.9153 & 0.8810 & 0.8606 & 0.7798 & 0.7366 & 0.7537 & 0.8212 \\
\hline & & NSPI & 0.9316 & 0.9086 & 0.8936 & 0.8364 & 0.7969 & 0.8198 & 0.8645 \\
\hline & & GNSPI & 0.9247 & 0.9000 & 0.8873 & 0.8370 & 0.7762 & 0.7952 & 0.8534 \\
\hline & & SSRBF & 0.9393 & 0.9180 & 0.9063 & 0.8543 & $\mathbf{0 . 8 1 0 7}$ & 0.8358 & 0.8774 \\
\hline \multirow{21}{*}{$\begin{array}{l}\text { L12 as } \\
\text { known } \\
\text { image }\end{array}$} & \multirow{7}{*}{ RMSE } & Gapfill & 0.0179 & 0.0222 & 0.0318 & 0.0592 & 0.0503 & 0.0462 & 0.0379 \\
\hline & & LLHM & 0.0116 & 0.0157 & 0.0259 & 0.0556 & 0.0447 & 0.0427 & 0.0327 \\
\hline & & GWR & 0.0116 & 0.0156 & 0.0257 & 0.0553 & 0.0445 & 0.0425 & 0.0325 \\
\hline & & WMLR & 0.0114 & 0.0152 & 0.0246 & 0.0507 & 0.0420 & 0.0407 & 0.0308 \\
\hline & & NSPI & 0.0103 & 0.0137 & 0.0209 & 0.0445 & 0.0368 & 0.0345 & 0.0268 \\
\hline & & GNSPI & 0.0105 & 0.0140 & 0.0215 & 0.0456 & 0.0386 & 0.0364 & 0.0278 \\
\hline & & SSRBF & $\mathbf{0 . 0 0 9 3}$ & 0.0126 & 0.0194 & 0.0425 & $\mathbf{0 . 0 3 5 4}$ & $\mathbf{0 . 0 3 3 0}$ & 0.0254 \\
\hline & \multirow{7}{*}{ CC } & Gapfill & 0.7241 & 0.6815 & 0.6882 & 0.6551 & 0.5212 & 0.6025 & 0.6454 \\
\hline & & LLHM & 0.8902 & 0.8494 & 0.7988 & 0.6808 & 0.6212 & 0.6567 & 0.7495 \\
\hline & & GWR & 0.8913 & 0.8511 & 0.8013 & 0.6845 & 0.6246 & 0.6602 & 0.7521 \\
\hline & & WMLR & 0.8947 & 0.8591 & 0.8199 & 0.7448 & 0.6762 & 0.6948 & 0.7816 \\
\hline & & NSPI & 0.9161 & 0.8902 & 0.8752 & 0.8107 & 0.7630 & 0.7929 & 0.8414 \\
\hline & & GNSPI & 0.9102 & 0.8823 & 0.8652 & 0.8019 & 0.7368 & 0.7663 & 0.8271 \\
\hline & & SSRBF & 0.9306 & 0.9060 & 0.8917 & 0.8291 & $\mathbf{0 . 7 8 3 5}$ & 0.8124 & 0.8589 \\
\hline & & Gapfill & 0.7163 & 0.6701 & 0.6766 & 0.6463 & 0.4996 & 0.5837 & 0.6321 \\
\hline & & LLHM & 0.8803 & 0.8322 & 0.7739 & 0.6412 & 0.5528 & 0.6018 & 0.7137 \\
\hline & & GWR & 0.8814 & 0.8340 & 0.7766 & 0.6451 & 0.5563 & 0.6056 & 0.7165 \\
\hline & UIQI & WMLR & 0.8852 & 0.8448 & 0.8030 & 0.7253 & 0.6342 & 0.6573 & 0.7583 \\
\hline & & NSPI & 0.9047 & 0.8751 & 0.8621 & 0.7993 & 0.7375 & 0.7717 & 0.8251 \\
\hline & & GNSPI & 0.9062 & 0.8754 & 0.8582 & 0.7935 & 0.7168 & 0.7474 & 0.8162 \\
\hline & & SSRBF & 0.9282 & 0.9015 & 0.8871 & 0.8209 & 0.7648 & 0.7974 & 0.8500 \\
\hline
\end{tabular}

Table 3 Classification accuracy for the filled results in Fig. 7 (the value in bold means the most accurate result in each case)

\begin{tabular}{|c|c|c|c|c|c|c|c|}
\hline & Gapfill & LLHM & GWR & WMLR & NSPI & GNSPI & SSRBF \\
\hline Water & 0.3577 & 0.4498 & 0.4525 & 0.5223 & 0.5625 & 0.5308 & $\mathbf{0 . 5 7 6 5}$ \\
\hline Vegetable 1 & 0.4703 & 0.6041 & 0.6049 & 0.5757 & 0.6073 & 0.6134 & $\mathbf{0 . 6 1 7 5}$ \\
\hline Vegetable 2 & 0.6901 & 0.5756 & 0.5808 & 0.6593 & 0.7732 & 0.7745 & $\mathbf{0 . 7 7 9 5}$ \\
\hline Vegetable 3 & 0.4177 & 0.5342 & 0.5353 & 0.5466 & 0.5116 & 0.5167 & $\mathbf{0 . 5 6 0 1}$ \\
\hline Buildings & 0.4658 & 0.4100 & 0.4142 & 0.4993 & 0.6257 & 0.6392 & $\mathbf{0 . 6 6 4 6}$ \\
\hline OA & 0.5344 & 0.5416 & 0.5447 & 0.5878 & 0.6522 & 0.6538 & $\mathbf{0 . 6 7 1 8}$ \\
\hline
\end{tabular}

We evaluated quantitatively the accuracies of the seven methods when L11 and L12 were used as the known image, respectively. The results based on root mean square error (RMSE), correlation coefficient (CC) and universal image quality index (UIQI) are shown in Table 2. Generally, compared with Gapfill, LLHM, GWR and WMLR, the RMSEs of the NSPI, GNSPI and SSRBF are smaller and their CCs and UIQIs are obviously larger. Moreover, SSRBF produces greater accuracies than NSPI and GNSPI for all six bands; that is, the accuracy of SSRBF is the greatest amongst the seven methods. Taking the results of using L12 as the known image as an example, the average CCs of the six bands of Gapfill, LLHM, GWR, WMLR, NSPI, GNSPI and SSRBF are 0.6454, 0.7495, $0.7521,0.7816,0.8414,0.8271$ and 0.8589 , respectively. The CC of SSRBF is $0.2135,0.1094,0.1068,0.0773,0.0175$ and
0.0318 larger than those of Gapfill, LLHM, GWR, WMLR, NSPI and GNSPI, respectively.

To further test the suitability of the predictions of the methods for application to land cover mapping, the unsupervised $k$-means clustering algorithm was adopted to classify the gap filling results in Fig. 7. Meanwhile, the reference land cover map was produced by $k$-means classification of the reference image in Fig. 7(i). For the classification result of filled pixels for each method, we calculated the producers' accuracy (PA) of five identified classes and the overall accuracy $(\mathrm{OA})$, as shown in Table 3. It is obvious that the classification accuracy of the SSRBF prediction is the greatest. This also indicates that the SSRBF prediction is the closest to the reference image. The McNemar's test on the classification results shows that there are significant differences between the classified maps of SSRBF and the other six methods. 


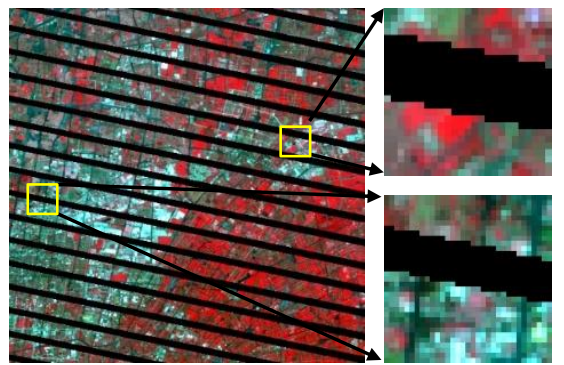

(a)

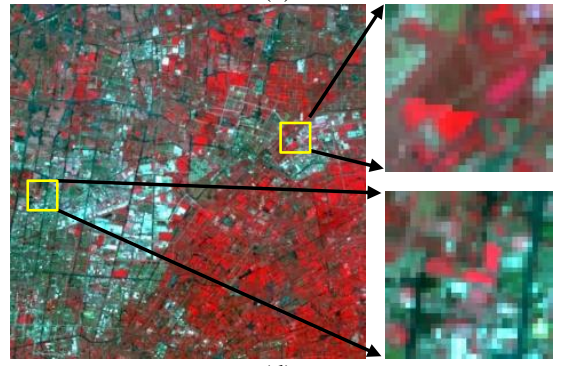

(d)

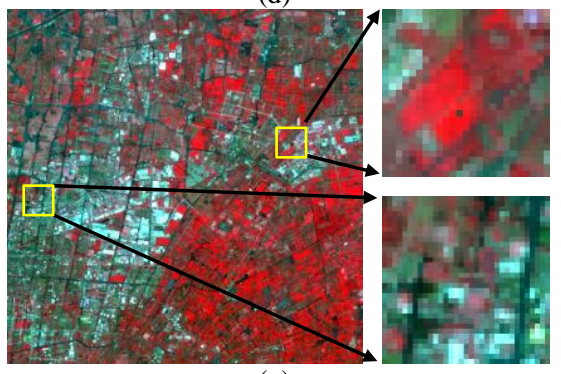

(g)

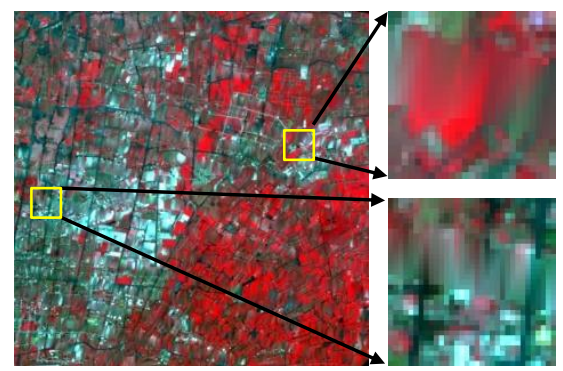

(b)

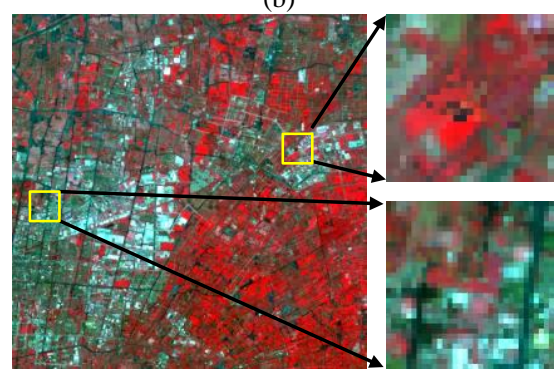

(e)

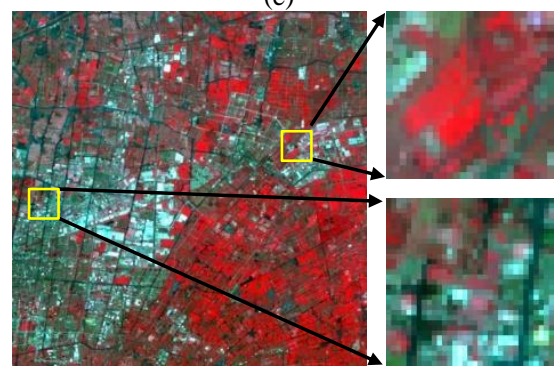

(h)

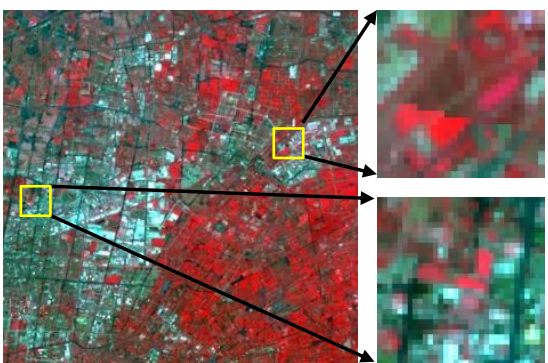

(c)

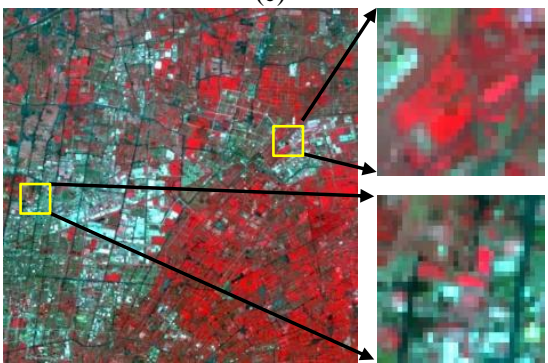

(f)

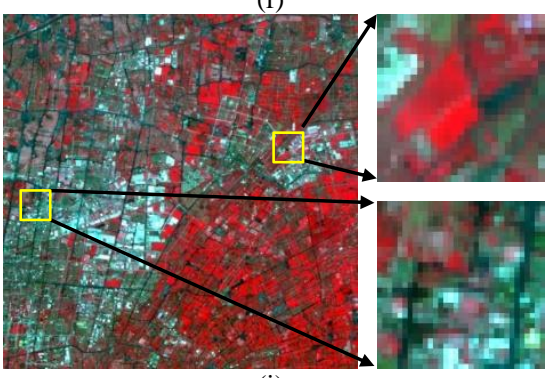

(i)

Fig. 12. Results of different methods for filling the gaps in the Zhejiang dataset (Region 2) acquired on April 22, 2016 (15 km by $15 \mathrm{~km}$ ) with two subareas shown on the right (NIR, red, and green bands as RGB). The image acquired on July 20, 2016 (L2) was used as the known image. (a) The simulated SLC-off image based on (i). (b) Gapfill. (c) LLHM. (d) GWR. (e) WMLR. (f) NSPI. (g) GNSPI. (h) SSRBF. (i) The actual image.

Table 4 Accuracies of the different gap filling methods in Zhejiang, China (Region 2), with L2 as known image (the values in bold and italic mean the most, and second most, accurate results in each case)

\begin{tabular}{|c|c|c|c|c|c|c|c|c|}
\hline & & Blue & Green & Red & NIR & SWIR1 & SWIR2 & Mean \\
\hline \multirow{7}{*}{ RMSE } & Gapfill & 0.0128 & 0.0137 & 0.0181 & 0.0461 & 0.0357 & 0.0405 & 0.0278 \\
\hline & LLHM & 0.0069 & 0.0086 & 0.0118 & 0.0464 & 0.0291 & 0.0276 & 0.0217 \\
\hline & GWR & 0.0069 & 0.0086 & 0.0118 & 0.0462 & 0.0288 & 0.0274 & 0.0216 \\
\hline & WMLR & 0.0060 & 0.0074 & 0.0103 & 0.0421 & 0.0242 & 0.0245 & 0.0191 \\
\hline & NSPI & 0.0078 & 0.0093 & 0.0121 & 0.0401 & 0.0238 & 0.0239 & 0.0195 \\
\hline & GNSPI & 0.0062 & 0.0075 & 0.0103 & 0.0368 & 0.0223 & 0.0239 & 0.0178 \\
\hline & SSRBF & 0.0065 & 0.0077 & 0.0105 & 0.0373 & 0.0216 & 0.0236 & 0.0179 \\
\hline \multirow{7}{*}{$\mathrm{CC}$} & Gapfill & 0.7504 & 0.7038 & 0.7014 & 0.5959 & 0.6150 & 0.6546 & 0.6702 \\
\hline & LLHM & 0.9283 & 0.8875 & 0.8782 & 0.5387 & 0.7492 & 0.8490 & 0.8051 \\
\hline & GWR & 0.9290 & 0.8887 & 0.8794 & 0.5456 & 0.7533 & 0.8510 & 0.8079 \\
\hline & WMLR & 0.9454 & 0.9166 & 0.9066 & 0.6508 & 0.8304 & 0.8818 & 0.8553 \\
\hline & NSPI & 0.9167 & 0.8807 & 0.8812 & 0.6982 & 0.8430 & 0.8900 & 0.8516 \\
\hline & GNSPI & 0.9435 & 0.9139 & 0.9084 & 0.7473 & 0.8596 & 0.8888 & 0.8769 \\
\hline & SSRBF & 0.9388 & 0.9107 & 0.9064 & 0.7395 & 0.8691 & 0.8934 & 0.8763 \\
\hline \multirow{7}{*}{ UIQI } & Gapfill & 0.7496 & 0.7017 & 0.6973 & 0.5874 & 0.6073 & 0.6465 & 0.6650 \\
\hline & LLHM & 0.9281 & 0.8868 & 0.8770 & 0.4765 & 0.7399 & 0.8456 & 0.7923 \\
\hline & GWR & 0.9288 & 0.8881 & 0.8783 & 0.4836 & 0.7444 & 0.8478 & 0.7952 \\
\hline & WMLR & 0.9445 & 0.9142 & 0.9040 & 0.6244 & 0.8213 & 0.8782 & 0.8478 \\
\hline & NSPI & 0.9156 & 0.8797 & 0.8809 & 0.6872 & 0.8421 & 0.8890 & 0.8491 \\
\hline & GNSPI & 0.9432 & 0.9133 & 0.9075 & 0.7367 & 0.8565 & 0.8870 & 0.8740 \\
\hline & SSRBF & 0.9388 & 0.9106 & 0.9061 & 0.7273 & 0.8669 & 0.8929 & 0.8738 \\
\hline
\end{tabular}

C. Simulation experiment on the Zhejiang data (Region 2; heterogeneous)

In the experiment on Region 2, S2 (acquired on April 22, 2016) was used to simulate the SLC-off image (shown in Fig. 12(a)) and L2 was used as the known image. The gap filling results of the seven methods are shown in Fig. 12. It is noticeable that the Gapfill result in Fig. 12(b) contains ambiguous stripes, which means that only using the valid pixels in the SLC-off image is insufficient for gap filling. Comparing 
Fig. 12(c) with Fig. 12(d), it is found that the results of LLHM and GWR are similar, in which the red objects are incorrectly predicted as gray in the first subarea. The WMLR and NSPI predictions in Fig. 12(e) and Fig. 12(f) look more satisfactory than Gapfill, LLHM and GWR, but for the red object in the first subarea, the WMLR and NSPI predictions contain some abrupt black and gray pixels, respectively. Checking the predictions of SSRBF and GNSPI, they are obviously more satisfactory and closer to the reference data.

The results of quantitative evaluation are shown in Table 4. From the three indices of each band, it is seen that compared to the other four methods, the RMSEs of GNSPI, NSPI and SSRBF are smaller and the CCs and UIQIs are larger. Moreover, the accuracy of SSRBF is greater than NSPI, and almost the same with GNSPI (the McNemar's test on the classification results suggest that there is no significant difference between SSRBF and GNSPI results).
D. Simulation experiment on the Northern New South Wales data (Region 3; abrupt change)

In the experiment on Region 3, S3 (acquired on December 12, 2004) was used to simulate the SLC-off image and L3 (acquired on November 26, 2004) was used as the known image. Fig. 13 shows the gap filling results of the seven methods. It can be seen from Fig. 13 that there are obvious gap areas in the predictions of the Gapfill, LLHM and GWR methods, especially at the edges of the two large red objects and the abrupt change area covered by the waterbody. For the other four methods, the differences between them can be observed clearly from the two subareas shown in Fig. 13(e)-(h). Moreover, it can be found that the SSRBF prediction is the closest to the actual image.

Table 5 lists the quantitative evaluation results of the seven methods. Comparing the three indices of each band, it is seen that the accuracy of SSRBF is the greatest, suggesting that SSRBF can produce more satisfactory results even in areas where abrupt changes occurred.

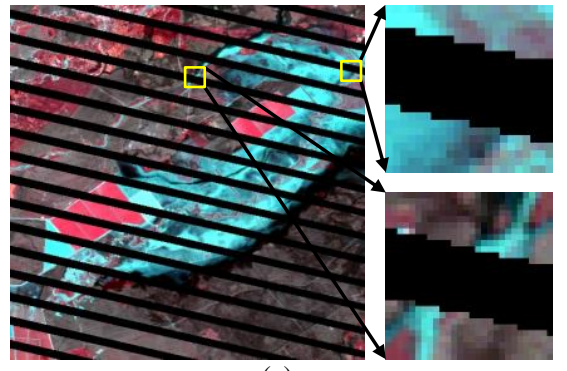

(a)

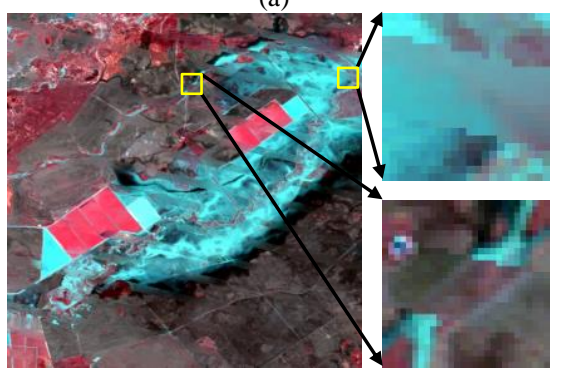

(d)

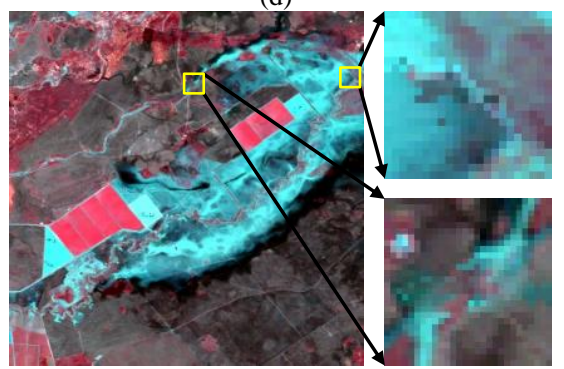

(g)

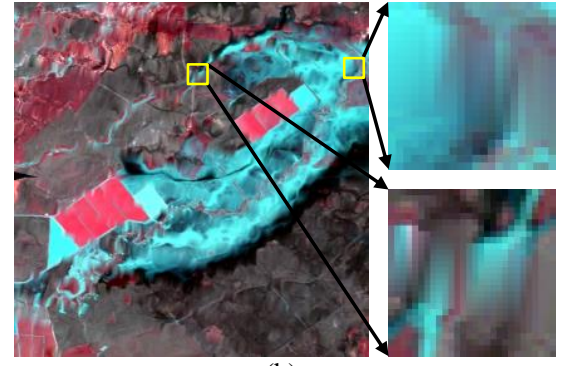

(b)

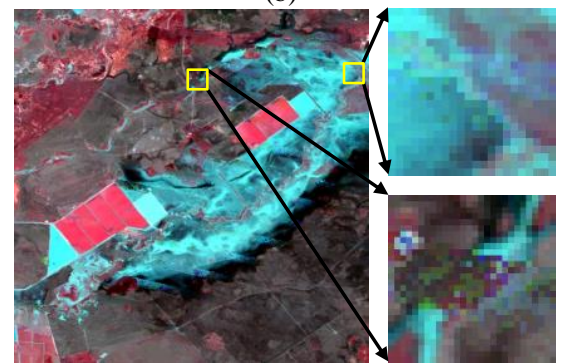

(e)

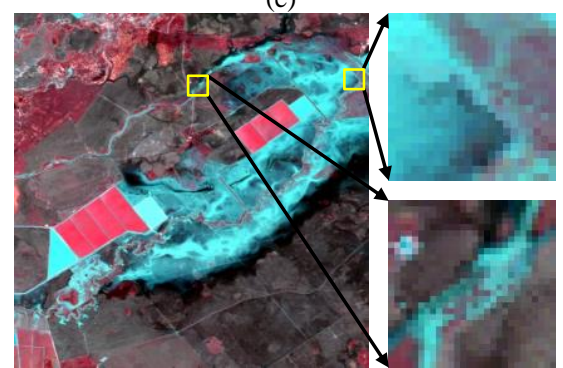

(h)

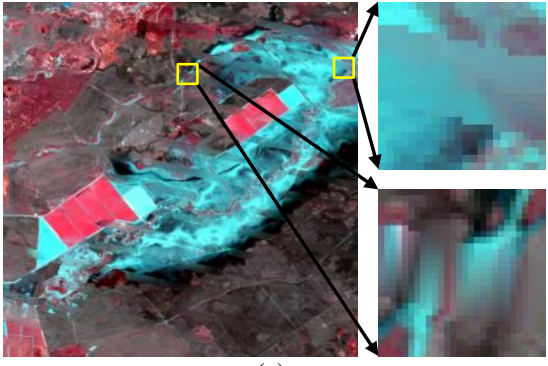

(c)

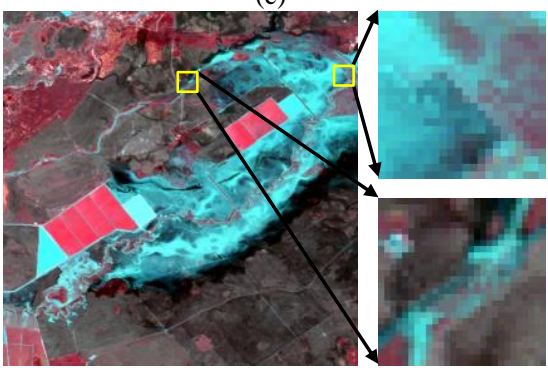

(f)

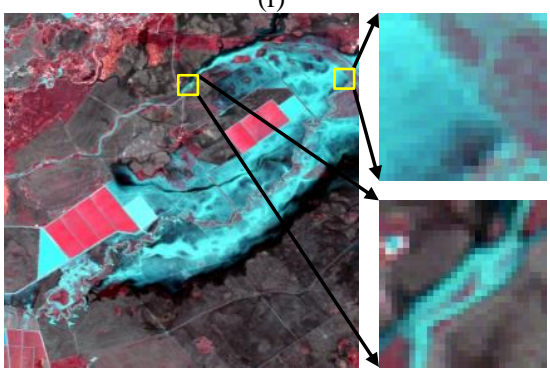

(i)

Fig. 13. Results of different methods for filling the gaps in the Northern New South Wales dataset (Region 3) acquired on December 12,2004 (15 km by $15 \mathrm{~km}$ ) with two subareas shown on the right (NIR, red, and green bands as RGB). The image acquired on November 26, 2004 (L3) was used as the known image. (a) The simulated SLC-off image based on (i). (b) Gapfill. (c) LLHM. (d) GWR. (e) WMLR. (f) NSPI. (g) GNSPI. (h) SSRBF. (i) The actual image.

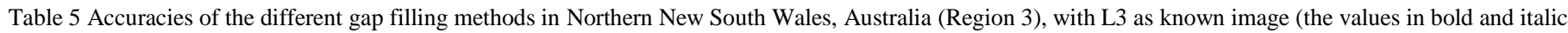
mean the most, and second most, accurate results in each case)

\begin{tabular}{|c|c|c|c|c|c|c|c|c|}
\hline & & Blue & Green & Red & NIR & SWIR1 & SWIR2 & Mean \\
\hline \multirow{6}{*}{ RMSE } & Gapfill & 0.0092 & 0.0135 & 0.0170 & 0.0206 & 0.0358 & 0.0273 & 0.0206 \\
\hline & LLHM & 0.0102 & 0.0154 & 0.0190 & 0.0234 & 0.0383 & 0.0281 & 0.0224 \\
\hline & GWR & 0.0101 & 0.0152 & 0.0188 & 0.0231 & 0.0379 & 0.0278 & 0.0222 \\
\hline & WMLR & 0.0108 & 0.0172 & 0.0193 & 0.0236 & 0.0363 & 0.0278 & 0.0225 \\
\hline & NSPI & 0.0087 & 0.0127 & 0.0153 & 0.0163 & 0.0320 & 0.0237 & 0.0181 \\
\hline & GNSPI & 0.0077 & 0.0111 & 0.0136 & 0.0172 & 0.0323 & 0.0268 & 0.0181 \\
\hline
\end{tabular}




\begin{tabular}{|c|c|c|c|c|c|c|c|c|}
\hline & SSRBF & $\mathbf{0 . 0 0 7 8}$ & $\mathbf{0 . 0 1 1 4}$ & $\mathbf{0 . 0 1 3 7}$ & $\mathbf{0 . 0 1 5 4}$ & $\mathbf{0 . 0 3 0 3}$ & $\mathbf{0 . 0 2 2 5}$ & $\mathbf{0 . 0 1 6 8}$ \\
\hline \multirow{6}{*}{} & Gapfill & 0.8922 & 0.8983 & 0.8837 & 0.9083 & 0.9031 & 0.8919 & 0.8963 \\
\cline { 2 - 9 } & LLHM & 0.8649 & 0.8634 & 0.8493 & 0.8801 & 0.8881 & 0.8850 & 0.8718 \\
\cline { 2 - 9 } & GWR & 0.8683 & 0.8671 & 0.8533 & 0.8832 & 0.8904 & 0.8874 & 0.8750 \\
\cline { 2 - 9 } & WMLR & 0.8477 & 0.8313 & 0.8475 & 0.8794 & 0.9002 & 0.8877 & 0.8656 \\
\cline { 2 - 9 } & NSPI & 0.9048 & 0.9095 & 0.9059 & 0.9438 & 0.9232 & 0.9190 & 0.9177 \\
\cline { 2 - 9 } & GNSPI & $\mathbf{0 . 9 2 5 9}$ & $\mathbf{0 . 9 3 1 2}$ & $\mathbf{0 . 9 2 6 0}$ & 0.9378 & 0.9222 & 0.8969 & 0.9233 \\
\cline { 2 - 9 } & SSRBF & 0.9226 & 0.9284 & 0.9252 & $\mathbf{0 . 9 4 9 9}$ & $\mathbf{0 . 9 3 1 2}$ & $\mathbf{0 . 9 2 7 8}$ & $\mathbf{0 . 9 3 0 8}$ \\
\hline \multirow{6}{*}{ UIQI } & Gapfill & 0.8903 & 0.8965 & 0.8817 & 0.9059 & 0.9009 & 0.8895 & 0.8941 \\
\cline { 2 - 9 } & LLHM & 0.8500 & 0.8497 & 0.8338 & 0.8701 & 0.8792 & 0.8756 & 0.8597 \\
\cline { 2 - 9 } & GWR & 0.8542 & 0.8540 & 0.8384 & 0.8735 & 0.8818 & 0.8783 & 0.8634 \\
\cline { 2 - 9 } & WMLR & 0.8438 & 0.8281 & 0.8438 & 0.8773 & 0.8969 & 0.8840 & 0.8623 \\
\cline { 2 - 9 } & NSPI & 0.9012 & 0.9064 & 0.9026 & 0.9423 & 0.9206 & 0.9158 & 0.9148 \\
\cline { 2 - 9 } & GNSPI & $\mathbf{0 . 9 2 5 2}$ & $\mathbf{0 . 9 3 0 5}$ & $\mathbf{0 . 9 2 5 1}$ & 0.9376 & 0.9208 & 0.8955 & 0.9225 \\
\cline { 2 - 9 } & SSRBF & 0.9204 & 0.9263 & 0.9229 & $\mathbf{0 . 9 4 8 6}$ & $\mathbf{0 . 9 2 9 3}$ & $\mathbf{0 . 9 2 5 7}$ & $\mathbf{0 . 9 2 8 9}$ \\
\hline
\end{tabular}

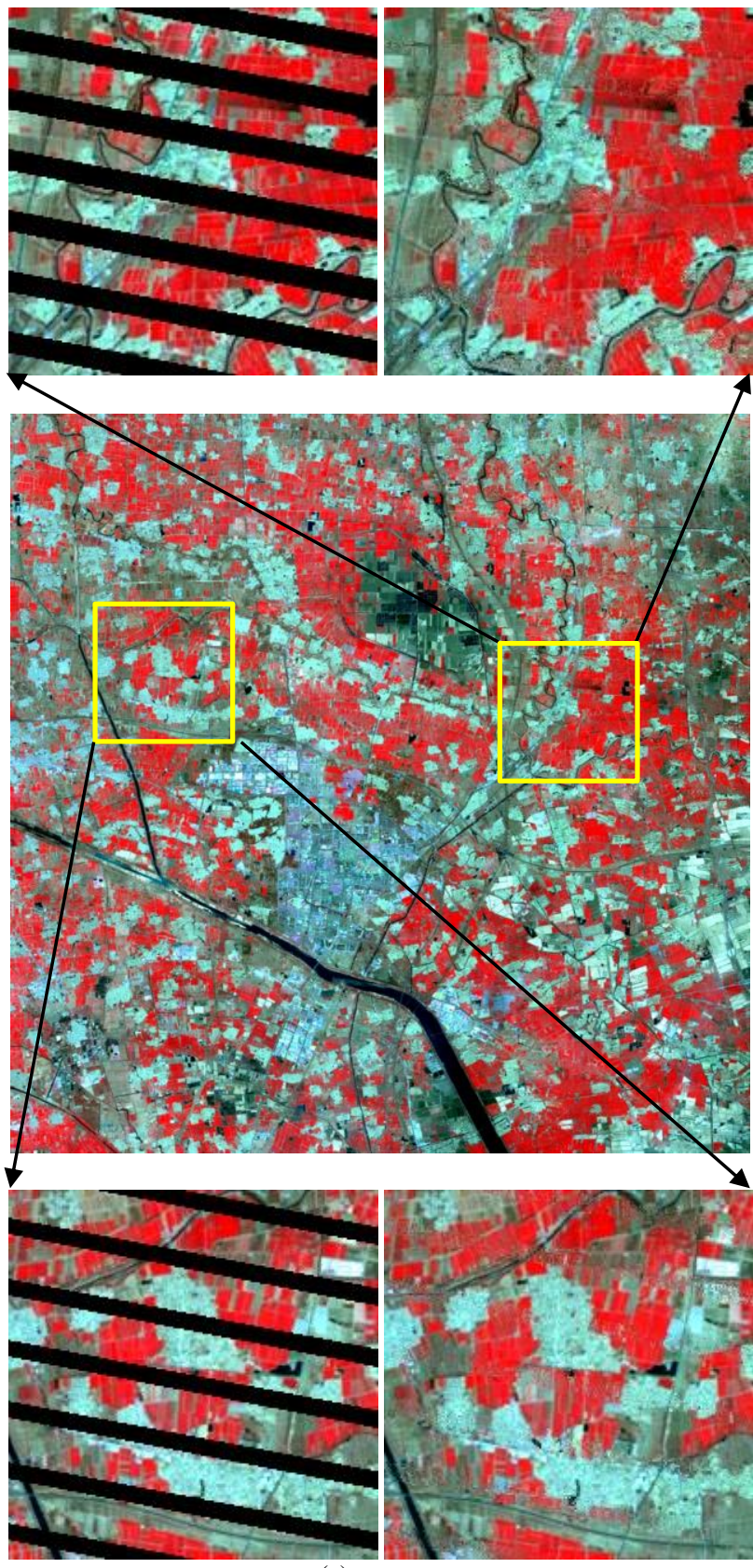

(a)

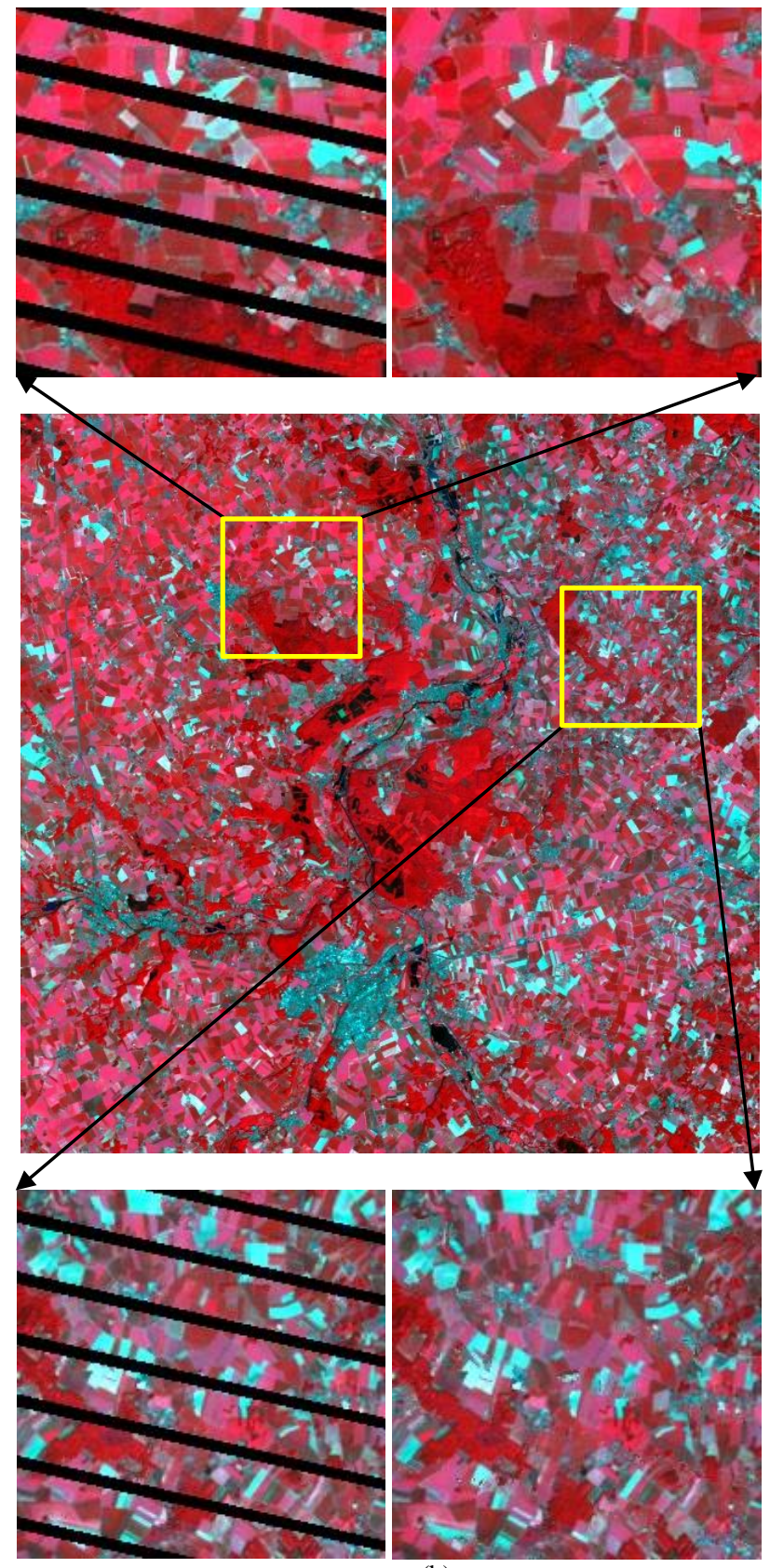

(b)

Fig. 14. Gap filling results of two real SLC-off images in Regions 4 and 5 (both $36 \mathrm{~km}$ by $36 \mathrm{~km}$; NIR, red and green bands as RGB), with two subareas and corresponding SLC-off areas zoomed. (a) Results for R4. (b) Results for R5.

Table 6 Accuracies of different schemes of using GLHM and spectral RBF (the value in bold means the most accurate result in each case) 


\begin{tabular}{|c|c|c|c|c|c|}
\hline & & $\begin{array}{c}\text { GLHM (No) } \\
\text { Spectral RBF (No) }\end{array}$ & $\begin{array}{c}\text { GLHM (No) } \\
\text { Spectral RBF (Yes) }\end{array}$ & $\begin{array}{c}\text { GLHM (Yes) } \\
\text { Spectral RBF (No) }\end{array}$ & $\begin{array}{c}\text { GLHM (Yes) } \\
\text { Spectral RBF (Yes) }\end{array}$ \\
\hline \multirow{3}{*}{$\begin{array}{c}\text { Region 1 } \\
\text { (L11 as known image) }\end{array}$} & RMSE & 0.0240 & 0.0235 & 0.0238 & $\mathbf{0 . 0 2 3 2}$ \\
\cline { 2 - 6 } & CC & 0.8761 & 0.8809 & 0.8785 & $\mathbf{0 . 8 8 3 4}$ \\
\cline { 2 - 6 } & UIQI & 0.8718 & 0.8752 & 0.8737 & $\mathbf{0 . 8 7 7 4}$ \\
\hline \multirow{2}{*}{ Region 1 } & RMSE & 0.0270 & 0.0260 & 0.0263 & $\mathbf{0 . 0 2 5 4}$ \\
\cline { 2 - 6 } (L12 as known image) & CC & 0.8403 & 0.8520 & 0.8484 & $\mathbf{0 . 8 5 8 9}$ \\
\cline { 2 - 6 } & UIQI & 0.8342 & 0.8429 & 0.8416 & $\mathbf{0 . 8 5 0 0}$ \\
\hline \multirow{2}{*}{$\begin{array}{c}\text { Region 2 } \\
\text { (L2 as known image) }\end{array}$} & RMSE & 0.0210 & 0.0205 & 0.0185 & $\mathbf{0 . 0 1 7 9}$ \\
\cline { 2 - 6 } & CC & 0.8374 & 0.8400 & 0.8695 & $\mathbf{0 . 8 7 6 3}$ \\
\cline { 2 - 6 } & UIQI & 0.8349 & 0.8381 & 0.8674 & $\mathbf{0 . 8 7 3 8}$ \\
\hline
\end{tabular}

E. Experiments on the Beijing and Versailles data (Regions 4 and 5; heterogeneous and abrupt change)

In this section, the proposed SSRBF-based gap filling method was applied to real Landsat 7 ETM+ SLC-off images for qualitative evaluation in practice. Specifically, for Regions 4 and 5, L4 (acquired on March 26, 2019) and L5 (acquired on August 7,2020 ) were used as the known images to restore the missing pixels in R4 (acquired on May 21, 2019) and R5 (acquired on May 27, 2020), respectively. The filled results of the two regions are shown in Fig. 14, with two magnified subareas and two corresponding SLC-off areas. The results show that the proposed SSRBF method can satisfactorily reconstruct the missing data in the SLC-off images in practice, even when there are distinct land cover changes between the known and SLC-off images (see, for example, the difference between the land cover in Region 5 in Fig. 6(a) and Fig. 6(b)).

\section{DISCUSSION}

\section{A. Benefits of using GLHM and the spectral RBF in SSRBF}

The proposed SSRBF-based gap filling method uses the pre-processing of GLHM and also integrates the spectral RBF with the existing spatial RBF. The rationality of the two parts is illustrated by experiments as follows. As before, $\mathrm{S} 1$ in Region 1 and S2 in Region 2 were used to simulate the SLC-off images and then the original complete images were used for quantitative evaluation. Accordingly, L11 and L12 in Region 1 and L2 in Region 2 were used as known images. Here, four different strategies are compared, and the average accuracies of the six bands are shown in Table 6. It can be seen from Table 6 that the accuracies of the results produced with GLHM are greater than without GLHM. For example, when using L2 as the known image for Region 2, the CCs of the spatial and spatial-spectral RBF increased by 0.0321 and 0.0363 after using GLHM. Moreover, the integration of the spectral RBF also increases the accuracy. For example, when using L12 as the known image for Region 1, the CC increased by 0.0117 with the use of the spectral RBF without GLHM, and increased by 0.0105 when using the spectral RBF with GLHM. In summary, either the GLHM or spectral RBF strategy can benefit the final result, and the accuracy can be further increased by integrating both.

GLHM not only alleviates the influence of temporal variation to a certain extent, but also facilitates the use of multiple known images. Specifically, GLHM establishes the relation between the known and SLC-off images through linear regression, which enables it to simultaneously utilize multiple known images through multiple regression. In multiple regression, the selection of the known images is flexible, by either using multiple Landsat OLI images or TM images, or even using both
Landsat OLI and TM images. It should be noted, however, that it is often the case in practice that only one image is close to the SLC-off image amongst the several known images. Thus, it is unclear how greatly the land cover changes that have occurred in other known images will impact the gap filling results. It is possible that the use of extra known images with a larger time interval may decrease the accuracy of the filled result. That is, the increase of input known images may not necessarily increase the accuracy of gap filling. On the other hand, intelligent models (e.g., the deep learning-based methods) can be developed potentially to take fuller advantage of the multiple known images by learning the trajectory of land cover change across time. Since multiple known images with high quality are usually difficult to acquire (or if available, are temporally distant, resulting in distinct land cover changes), this paper considers the general case of using one known image.

\section{B. Advantages of the proposed SSRBF-based gap filling method}

Table 7 Running times (in units of seconds) of the methods for Regions 1 and 2

\begin{tabular}{|c|c|c|}
\hline & Region 1 & Region 2 \\
\hline LLHM & 192.4 & 353.9 \\
\hline GWR & 1909.0 & 2006.2 \\
\hline WMLR & 113.7 & 141.0 \\
\hline NSPI & 25.5 & 28.5 \\
\hline GNSPI & 464.1 & 447.7 \\
\hline SSRBF & 49.2 & 58.0 \\
\hline
\end{tabular}

The gap filling method proposed in this paper holds the following three advantages. First, SSRBF is an accurate interpolation method with a simple mathematical calculation model, which means that it has a low computational complexity. SSRBF interpolation establishes a clear analytic function between the predicted value and the known values. This process does not require any auxiliary information, and the solution is fast and simple to realize, which is conducive to its widespread application in various scenarios. The running times of the methods for Regions 1 and 2 are shown in Table 7 (CPU: Intel Xeon Silver 4110). It is seen that the computing time of SSRBF is very close to that of NSPI and is significantly less than for other methods. Second, compared with the conventional spatial RBF, the integration of spectral RBF enables SSRBF to characterize the difference between similar pixels more accurately and achieve greater non-linear fitting capability, as shown in the results in Section IV-A. Third, the GLHM process applied to the known images can, to a certain extent, reduce the impact of apparent differences between the known and SLC-off images in feature space due to the acquisition time interval and land cover changes, as proved in the Section II-A. GLHM, thus, extends the application utility of SSRBF and enables it to deal 
with the challenging case where large differences exist between the known and SLC-off images.

\section{Temporal distance between the known and SLC-off images}

Since GLHM in the proposed SSRBF method is used to reduce the difference between the SLC-off and known images caused by temporal changes, it is necessary to investigate how greatly the temporal distance influences the gap filling accuracy after GLHM. The Region 1 dataset was used for testing. Based on the results for Regions 1-3 in Section III, it was found that the performances of NSPI and GNSPI are generally comparable. Note, however, that in the experiment for Region 1 in Section III- $B$, NSPI performs more satisfactorily than GNSPI. Therefore, NSPI was selected for further comparison with SSRBF in this section.

In Section III- $B$, it was shown that using L12 that was acquired further from the SLC-off image leads to a lower accuracy of gap filling than for L11. To explore the effects of time interval on the accuracies of the results, we conducted experiments using different OLI data in Region 1 over two years (between 2016 and 2018) as the known image for gap filling of the SLC-off image simulated based on S1 (acquired on August 27, 2016). Fig. 15 shows the accuracies (in terms of CC) in relation to the known images at different times. In Fig.15, the three horizontal dotted lines from left to right represent the dates of August 27, 2016, August 27, 2017, and August 27, 2018, respectively. From Fig. 15, three interesting points can be observed. First, the accuracies of gap filling using both methods show a periodic change between years. Specifically, in the same cycle (e.g., from August 27, 2016 to August 27, 2017), as the acquisition date of the known image moves away from the SLC-off image and gradually approaches the same date in the next year, the gap filling accuracy shows a trend of first decreasing and then increasing. The reason is that within one year, due to periodic seasonal changes, the difference between the known and SLC-off images in hue first reaches the maximum in the opposite season in the same year and then the minimum in the same season in the next year. Furthermore, for the three dates that are closest to the three horizontal dotted lines (i.e., the same day of the year with the SLC-off image), the accuracies are the greatest amongst all the results. Second, taking a year as a cycle, the accuracies of the filled results decrease year-by-year. For example, the largest CC decreases by 0.03 from 2016 to 2017 , and by 0.04 from 2017 to 2018 . This is because more land cover changes such as abrupt changes occur when the temporal distance to the SLC-off image increases, leading to greater uncertainty in gap filling. Third, SSRBF consistently performs more satisfactorily and stably than NSPI.

Based on the above observations, two further conclusions can be drawn. First, the differences in hue caused by seasonal changes and land cover changes (e.g., abrupt changes) between the known and SLC-off images have a significant influence on the accuracies of the filled results. Second, SSRBF is less affected by seasonal changes than the existing NSPI. This means that in the proposed SSRBF-based gap filling method, GLHM can reduce the influence of hue differences to a certain extent. Based on the assumption of very few land cover changes, if a known image is acquired far from the SLC-off image in time, but is closer to the SLC-off image in hue, then the gap filling results may be more satisfactory than using another known image that is closer to the SLC-off image in time, but significantly different in hue. Therefore, the acceptable time interval for a useful known image depends mainly on whether there are sufficiently small differences in hue and land cover changes between it and the SLC-off image. In the future, further case studies may be needed to investigate how large hue differences and land cover changes (especially abrupt changes) may have an unacceptable negative impact on the use of known images.

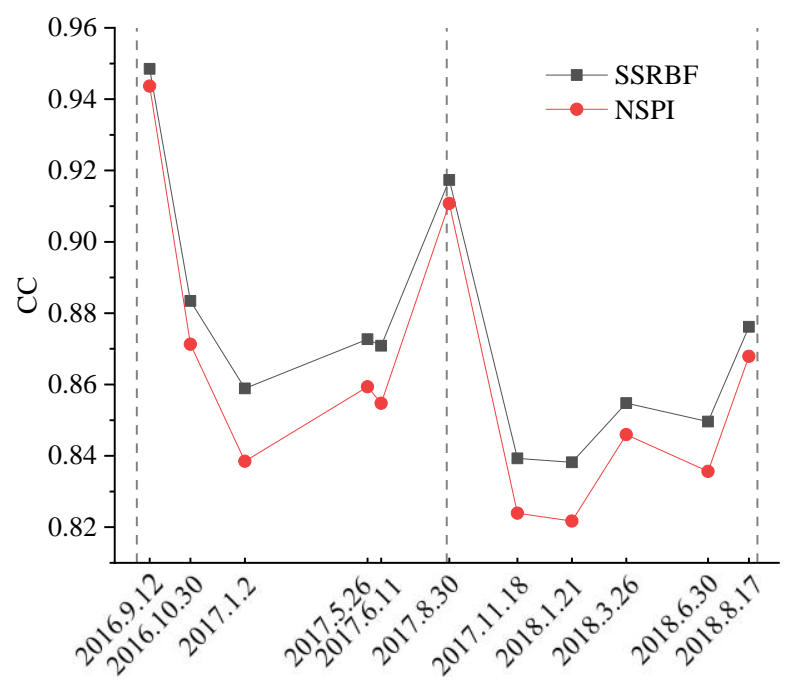

Fig. 15. CCs of NSPI and SSRBF for gap filling of the S1-simulated SLC-off image (on August 27, 2016) using different known images acquired between August 1, 2016 and September 1, 2018 (the three horizontal dotted lines mark the dates of August 27, 2016, August 27, 2017 and August 27, 2018).

\section{Generalization of SSRBF}

Although the SSRBF method is proposed for SLC-off image gap filling in this paper, its application is not limited to this specific issue. Essentially, the principle of gap filling for SLC-off images is similar to other data reconstruction issues such as cloud removal and infilling [11], [42], [43], all of which are performed by borrowing information from the spatially and temporally neighboring data of the gap image. Moreover, besides optical images, there are also missing data in a variety of quantitative products, such as global soil moisture [44], [45], land surface temperature (LST) [46], [47] and the normalized difference vegetation index (NDVI) [48], [49], etc. Given the competitive performance of SSRBF in this paper, it has great potential for other data reconstruction problems. In future research, the feasibility of SSRBF will be examined for other data reconstruction problems, and the model will also be further extended for tackling challenges in specific problems.

\section{CONCLUSION}

Landsat 7 ETM+ data play a crucial role in various applications, even after the SLC failure. Therefore, it is of great significance to fill gaps in the SLC-off images. To this end, we developed the SSRBF method for gap filling in this paper. SSRBF uses GLHM as a pre-processing step and, further, considers spectral information in characterizing the relation between pixels in addition to the conventional spatial-only-based RBF. Through the case studies in five different regions, it is shown that the proposed SSRBF method is an accurate solution for gap filling. Either the GLHM or 
spectral RBF strategy are beneficial to the prediction, and the accuracy is further increased by integrating both aspects. Compared with the existing Gapfill, LLHM, GWR, WMLR, NSPI and GNSPI methods, SSRBF produces consistently greater accuracy in gap filling.

The SSRBF method proposed in this paper are not only limited to the issue of Landsat 7 ETM+ SLC-off images gap filling, but are also applicable to other data reconstruction issues such as cloud removal and infilling, and this will be investigated in future research.

\section{REFERENCES}

[1] T. Arvidson, S. Goward, J. Gasch, D. Williams, "Landsat-7 long-term acquisition plan: development and validation," Photogrammetric Engineering and Remote Sensing, vol. 72, pp. 1137-1146. 2006.

[2] F. Chen, X. Zhao, H. Ye, Data Acquisition Applications. Edited by Gray, P. R., Wooley, B. A., Brodersen, R. W., Berkeley, CA, United States. 2012.

[3] M. A. Wulder, J. C. White, T. R. Loveland, C. E. Woodcock, A. S. Belward, W. B. Cohen, E. A. Fosnight, J. Shaw, J. G. Masek, D. P. Roy, "The global Landsat archive: Status, consolidation, and direction," Remote Sensing of Environment, vol. 185, pp. 271-283, 2016.

[4] M. A. Wulder, T. R. Loveland, D. P. Roy, C. J. Crawford, J. G. Masek, C. E. Woodcock, R. G. Allen, M. C. Anderson, A. S. Belward, W. B. Cohen, J. Dwyer, A. Erb, F. Gao, P. Griffiths, D. Helder, T. Hermosilla, J. D. Hipple, P. Hostert, M. J. Hughes, J. Huntington, D. M. Johnson, R. Kennedy, A. Kilic, Z. Li, L. Lymburner, J. McCorkel, N. Pahlevan, T. A. Scambos, C. Schaaf, J. R. Schott, Y. Sheng, J. Storey, E. Vermote, J. Vogelmann, J. C. White, R. H. Wynne, Z. Zhu, "Current status of Landsat program, science, and applications," Remote Sensing of Environment, vol. 225, pp. 127-147, 2019.

[5] Z. Zhu, M. A. Wulder, D. P. Roy, C. E. Woodcock, M. C. Hansen, V. C. Radeloff, S. P. Healey, C. Schaaf, P. Hostert, P. Strobl, J. Pekel, L. Lymburner, N. Pahlevan, T. A. Scambos, "Benefits of the free and open Landsat data policy," Remote Sensing of Environment, vol. 224, pp. 382-385, 2019.

[6] C. E. Woodcock, R. Allen, M. Anderson, A. Belward, R. Bindschadler, W. Cohen, F. Gao, S. N. Goward, D. Helder, E. Helmer, R. Nemani, L. Oreopoulos, J. Schott, P. S. Thenkabail, E. F. Vermote, J. Vogelmann, M. A. Wulder, R. Wynne, "Free access to Landsat imagery," Science, vol. 320, pp. 1011, 2008.

[7] D. Williams, S. Goward, T. Arvidson, "Landsat: yesterday, today, and tomorrow," Photogrammetric Engineering and Remote Sensing, vol. 72, no. 10, pp. 1171-1178, 2006.

[8] M. A. Wulder, J. C. White, S. N. Goward, J. G. Masek, J. R. Irons, M. Herold, W. B. Cohen, T. R. Loveland, C. E. Woodcock, "Landsat continuity: issues and opportunities for land cover monitoring," Remote Sensing of Environment, vol. 112, no. 3, pp. 955-969, 2008.

[9] M. A. Wulder, J. G. Masek, W. B. Cohen, "Opening the archive: how free data has enabled the science and monitoring promise of Landsat," Remote Sensing of Environment, vol. 122, pp. 2-10, 2012.

[10] USGS, 2004. "Phase 2 gap-fill algorithm: SLC-off gap-filled products gap-fill algorithm methodology," Landsat.usgs.gov/documents/L7SLCGapFilledMethod.pdf (Available online at (accessed 28 November 2010)).

[11] H. Shen, X. Li, Y. Qian, L. Zhang, Q. Yuan, "An effective thin cloud removal procedure for visible remote sensing images," ISPRS Journal of Photogrammetry and Remote Sensing, vol. 96, pp. 224-235, 2014.

[12] H. Shen, X. Li, Q. Cheng, C. Zeng, G. Yang, H. Li, L. Zhang, "Missing information reconstruction of remote sensing data: a technical review," IEEE Geoscience and Remote Sensing Magazine, vol. 3, no. 3, pp. 61-85, 2015.

[13] Z. Zhu, C. E. Woodcock, P. Olofsson, "Continuous monitoring of forest disturbance using all available Landsat imagery," Remote Sensing of Environment, vol. 122, pp. 75-91, 2012.

[14] L. Malambo, C. D. Heatwole, "A multitemporal profile-based interpolation method for gap filling nonstationary data," IEEE Transactions on geoscience and remote sensing, vol. 54, no. 1, pp. 252-261, 2016

[15] M. E. Romero-Sanchez, R. Ponce-Hernandez, S. E. Franklin, C. A. Aguirre-Salado, "Comparison of data gap-filling methods for Landsat ETM+ SLC-off imagery for monitoring forest degradation in a semi-deciduous tropical forest in Mexico," International Journal of Remote Sensing, vol. 36, no. 11, pp. 2786-2799, 2015.

[16] D. P. Roy, J. Ju, P. Lewis, C. Schaaf, F. Gao, M. Hansen, E. Lindquist, "Multi-temporal MODIS-Landsat data fusion for relative radiometric normalization, gap filling, and prediction of Landsat data," Remote Sensing of Environment, vol. 112, no. 6, pp. 3112-3130, 2008.

[17] M. M. Reza, S. N. Ali, "Using IRS products to recover Landsat7 ETM+ defective images," American Journal of Applied Sciences, vol. 5, no. 6, pp. 618-625, 2008.

[18] A. D. Boloorani, S. Erasmi, M. Kappas, "Multi-source image reconstruction: exploitation of EO-1/ALI in Landsat-7/ETM+ SLC-off gap filling," Journal of Optical Microsystems, vol. 6812, pp. 681219, 2008.

[19] F. Chen, L. Tang, Q. Qiu, "Exploitation of CBERS-02B as auxiliary data in recovering the Landsat7 ETM+ SLC-off image," In Proceedings of the 18 International Conference on Geoinformatics. Peking University, Beijing, China, 2010.

[20] C. Zhang, W. Li, D. Civco, "Application of geographically weighted regression to fill gaps in SLC-off Landsat ETM+ satellite imagery," International Journal of Remote Sensing, vol. 35, no. 22, pp. 7650-7672, 2014.

[21] C. Zeng, H. Shen, L. Zhang, "Recovering missing pixels for Landsat ETM+ SLC-off imagery using multi-temporal regression analysis and a regularization method," Remote Sensing of Environment, vol. 131, pp. 182-194, 2013.

[22] S. K. Maxwell, G. L. Schmidt, J. C. Storey, "A multi-scale segmentation approach to filling gaps in Landsat ETM+ SLC-off images," International Journal of Remote Sensing, vol. 28, no. 23, pp. 5339-5356, 2007.

[23] F. Bédard, G. Reichert, R. Dobbins, I. Trépanier, "Evaluation of segment-based gap-filled Landsat ET M+ SLC-off satellite data for land cover classification in southern Saskatchewan, Canada," International Journal of Remote Sensing, vol. 29, no. 7, pp. 2041-2054, 2008.

[24] C. Zhang, W. Li, D. Travis, "Gaps-fill of SLC-off Landsat ETM plus satellite image using a geostatistical approach," International Journal of Remote Sensing, vol. 28, no. 22, pp. 5103-5122, 2007.

[25] M. J. Pringle, M. Schmidt, J. S. Muir, "Geostatistical interpolation of SLC-off Landsat ETM plus images," ISPRS Journal of Photogrammetry and Remote Sensing, vol. 64, no. 6, pp. 654-664, 2009.

[26] J. Chen, X. Zhu, J. E. Vogelmann, F. Gao, S. Jin, "A simple and effective method for filling gaps in Landsat ETM+ SLC-off images," Remote Sensing of Environment, vol. 115, no. 4, pp. 1053-1064, 2011.

[27] X. Zhu, D. Liu, J. Chen, "A new geostatistical approach for filling gaps in Landsat ETM+ SLC-off images," Remote Sensing of Environment, vol. 124, pp. 49-60, 2012.

[28] Y. Luo, K. Guan, J. Peng, "STAIR: A generic and fully-automated method to fuse multiple sources of optical satellite data to generate a high-resolution, daily and cloud-/gap-free surface reflectance product," Remote Sensing of Environment, vol. 214, pp. 87-99, 2018.

[29] G. Yin, G. Mariethoz, M. F. McCabe, "Gap-filling of Landsat 7 imagery using the direct sampling method," Remote Sensing, vol. 9, no. 1, pp. 12-21, 2017.

[30] Q. Yuan, H. Shen, T. Li, Z. Li, S. Li, Y. Jiang, H. Xu, W. Tan, Q. Yang, J. Wang, J. Gao, L. Zhang, "Deep learning in environmental remote sensing: achievements and challenges," Remote Sensing of Environment, vol. 241, pp. 111716, 2020.

[31] Q. Zhang, Q. Yuan, C. Zeng, X. Li, Y. Wei, "Missing data reconstruction in remote sensing image with a unified spatial-temporal-spectral deep convolutional neural network," IEEE Transactions on Geoscience and Remote Sensing, vol. 56, no. 8, pp. 4274-4288, 2018.

[32] Y. J. Lee, J. Yoon, "Nonlinear image upsampling method based on radial basis function interpolation," IEEE Transactions on Image Processing, vol. 19, no. 10, pp. 2682-2692, 2010.

[33] F. Magoules, L. A. Diago, I. Hagiwara, "Efficient preconditioning for image reconstruction with radial basis functions," Advances in Engineering Software, vol. 38, pp. 320-327, 2007.

[34] M. D. Buhmann, "Radial basis functions: theory and implementations," Cambridge, UK: Cambridge University Press. 2003.

[35] R. Pollandt, "Solving nonlinear differential equations of mechanics with the boundary element method and radial basis functions," International Journal for Numerical Methods in Engineering, vol. 40, no. 1, pp. 61-73, 1997.

[36] C. E. Torres, L. A. Barba, "Fast radial basis function interpolation with Gaussians by localization and iteration," Journal of Computational Physics, vol. 228, pp. 4976-4999, 2009. 
[37] S. Wang, K. Lim, B. Khoo, M. Wang, "An extended level set method for shape and topology optimization," Journal of Computational Physics, vol. 221, no. 1, pp. 395-421, 2007.

[38] Q. Wang, W. Shi, P. M. Atkinson, "Sub-pixel mapping of remote sensing images based on radial basis function interpolation," ISPRS Journal of Photogrammetry and Remote Sensing, vol. 92, pp. 1-15, 2014.

[39] R. Cao, Y. Chen, J. Chen, X. Zhu, M. Shen, "Thick cloud removal in Landsat images based on autoregression of Landsat time-series data," Remote Sensing of Environment, vol. 249, pp. 112001, 2020.

[40] T. Hu, X. Huang, J. Li, L. Zhang, "A novel co-training approach for urban land cover mapping with unclear Landsat time series imagery," Remote Sensing of Environment, vol. 217, pp. 144-157, 2018.

[41] A. Sadiq, L. Edwar, G. Sulong, "Recovering the large gaps in Landsat 7 SLC-off imagery using weighted multiple linear regression (WMLR)," Arabian Journal of Geosciences, vol. 10, no. 18, pp. 403, 2017.

[42] B. Chen, B. Huang, L. Chen, B. Xu, "Spatially and temporally weighted regression: a novel method to produce continuous cloud-free Landsat imagery," IEEE Transactions on Geoscience and Remote Sensing, vol 55, no. 1, pp. 27-37, 2016.

[43] M. Xu, X. Jia, M. Pickering, A. J. Plaza, "Cloud removal based on sparse representation via multitemporal dictionary learning" IEEE Transactions on Geoscience \& Remote Sensing, vol. 54, no. 5, pp. 2998-3006, 2016.

[44] M. M. Al-Khaldi, J. T. Johnson, A. J. O’Brien, A. Balenzano, F. Mattia, "Time-series retrieval of soil moisture using CYGNSS," IEEE Transactions on Geoscience and Remote Sensing, vol. 57, no. 7, pp. 4322-4331, 2019.

[45] C. S. Kang, K. D. Kanniah, Y. H. Kerr, "Calibration of SMOS soil moisture retrieval algorithm: A case of tropical site in Malaysia," IEEE Transactions on Geoscience and Remote Sensing, vol. 57, no. 6, pp. 3827-3839, 2019.

[46] N. K. Malakar, G. C. Hulley, S. J. Hook, K. Laraby, M. Cook and J. R. Schott, "An operational land surface temperature product for Landsat thermal data: Methodology and validation," IEEE Transactions on Geoscience and Remote Sensing, vol. 56, no. 10, pp. 5717-5735, 2018.

[47] H. Li, R. Li, Y. Yang, B. Cao, Z. Bian, T. Hu, Y. Du, L. Sun, Q. Liu, "Temperature-based and radiance-based validation of the collection 6 MYD11 and MYD21 land surface temperature products over barren surfaces in northwestern China," IEEE Transactions on Geoscience and Remote Sensing, 2020.

[48] A. F. Militino, M. D. Ugarte, U. Pérez-Goya, M. G. Genton, "Interpolation of the mean anomalies for cloud filling in land surface temperature and normalized difference vegetation index," IEEE Transactions on Geoscience and Remote Sensing, vol. 57, no. 8, pp. 6068-6078, 2019.

[49] X. Tian, M. Zhang, C. Yang, J. Ma, "FusionNDVI: A computational fusion approach for high-resolution normalized difference vegetation index," IEEE Transactions on Geoscience and Remote Sensing, 2020.

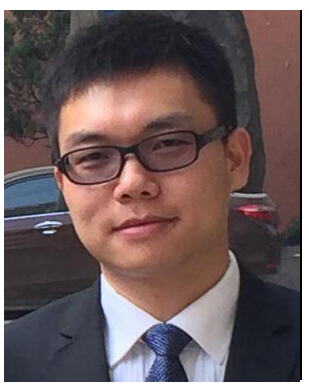

Qunming Wang received the Ph.D. degree from the Hong Kong Polytechnic University, Hong Kong, in 2015.

$\mathrm{He}$ is currently a Professor with the College of Surveying and Geo-Informatics, Tongji University, Shanghai, China. He was a Lecturer (Assistant Professor) with Lancaster Environment Centre, Lancaster University, Lancaster, U.K., from 2017 to 2018. His 3-year Ph.D. study was supported by the hypercompetitive Hong Kong Ph.D. Fellowship and his Ph.D. thesis was awarded as the Outstanding Thesis in the Faculty. He has authored or coauthored over 50 peer-reviewed articles in international journals such as Remote Sensing of Environment, IEEE Transactions on Geoscience and Remote Sensing, and ISPRS Journal of Photogrammetry and Remote Sensing. His research interests include remote sensing, image processing, and geostatistics.

Dr. Wang serves as Associate Editor for Science of Remote Sensing (sister journal of Remote Sensing of Environment) and Photogrammetric Engineering \& Remote Sensing, and was Associate Editor for Computers and Geosciences (2017-2020).

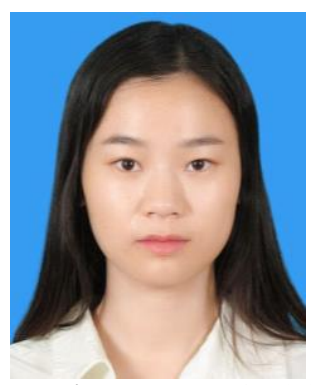

Lanxing Wang received the B.S. degree from Changsha University of Science \& Technology, Changsha, China, in 2019. She is currently working toward the M.S. degree at Tongji University, Shanghai, China. Her research interests include remote sensing data reconstruction.

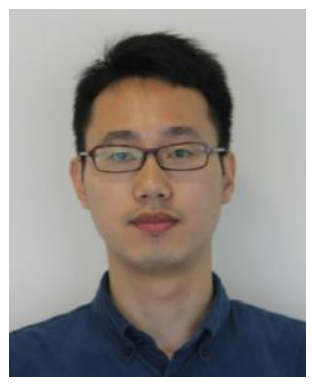

Zhongbin Li received the Ph.D. degree in remote sensing and geographic information science from The Hong Kong Polytechnic University, Hong Kong, in 2016.

He is currently an Assistant Professor at Center for Global Change and Earth Observations, Michigan State University, MI, USA. He was a Post-Doctoral Research Fellow at the Geospatial Sciences Center of Excellence, South Dakota State University, Brookings, SD, USA between July 2016 and May 2019. In 2015, he was a visiting Ph.D. student with the School of Geographical Sciences and Urban Planning, Arizona State University, Tempe, AZ, USA. His research interests include global change, remote sensing, and petabyte satellite data processing.

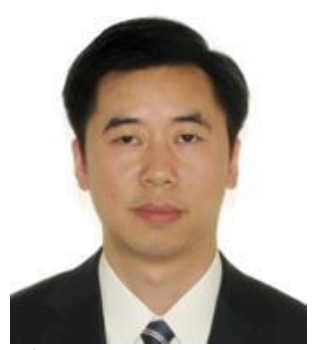

Xiaohua Tong received the Ph.D. degree in traffic engineering from Tongji University, Shanghai, China, in 1999.

$\mathrm{He}$ is currently a Professor with the College of Surveying and Geoinformatics, Tongji University. He was a Research Fellow with Hong Kong Polytechnic University, Hong Kong, in 2006, and a Visiting Scholar with the University of California, Santa Barbara, CA, USA, between 2008 and 2009. His research interests include remote sensing, geographic information system, uncertainty and spatial data quality, and image processing for high-resolution and hyperspectral images.

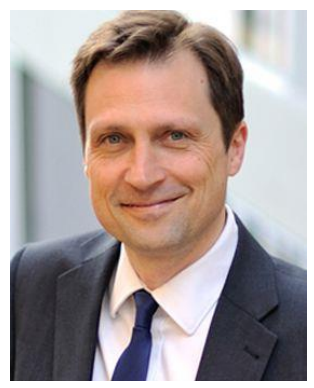


Peter M. Atkinson received the Ph.D. degree from the University of Sheffield (NERC CASE award with Rothamsted Experimental Station) in 1990. More recently, he received the MBA degree from the University of Southampton in 2012.

He is currently Distinguished Professor of Spatial Data Science and Dean of the Faculty of Science and Technology at Lancaster University, UK. He was previously Professor of Geography at the University Southampton, where he is currently Visiting Professor. He is also Visiting Professor at the Chinese Academy of Sciences, Beijing. He previously held the Belle van Zuylen Chair at Utrecht University, the Netherlands, is a recipient of the Peter Burrough Award of the International Spatial Accuracy Research Association and is a Fellow of the Learned Society of Wales. The main focus of his research is in remote sensing, geographical information science and spatial (and space-time) statistics applied to a range of environmental science and socio-economic problems. $\mathrm{He}$ has published over 300 peer-reviewed articles in international scientific journals and around 50 refereed book chapters. He has also edited nine journal special issues and eight books.

Professor Atkinson is Editor-in-Chief of Science of Remote Sensing, a sister journal of Remote Sensing of Environment. He also sits on the editorial boards of several further journals including Geographical Analysis, Spatial Statistics, International Journal of Applied Earth Observation and Geoinformation, and Environmental Informatics. 